\title{
Catastrophic failure in planar landslides with a fully softened weak zone
}

\author{
W. ZHANG*, D. WANG*, M. F. RANDOLPH* and A. M. PUZRIN $\dagger$
}

\begin{abstract}
Catastrophic landslides pose significant threats to life and property, and in the case of submarine landslides damage to offshore infrastructure. Although widely discussed, the triggering mechanisms and propagation criteria for catastrophic failure remain open issues. This study investigates a particular case of shear band initiation history: creation of a fully softened initial failure zone in a thin lens of a weaker material causing catastrophic failure of an infinite planar slope in sensitive clay under undrained conditions. The corresponding shear band propagation criteria were derived analytically using a process zone approach and validated numerically using a static large-deformation finite-element method. New analytical solutions taking account of elastic deformations within the shear band and in the entire sliding layer are established for both a linear strength degradation curve and an exponential strength degradation curve. Advantages of formulating propagation criteria in terms of the critical length of the fully softened initial failure zone (excluding process zones) are demonstrated, with the more realistic exponential degradation case producing a more stringent criterion than the linear degradation case.
\end{abstract}

KEYWORDS: failure; finite-element modelling; landslides; offshore engineering; shear strength; slopes

\section{INTRODUCTION}

Subaerial and submarine landslides pose a significant hazard in many parts of the world, potentially resulting in loss of life and property or, in the case of submarine landslides, damage to offshore infrastructure or generation of tsunamis. In sensitive soil deposits, loss of strength during shearing can cause rapid escalation of the scale of the landslide.

There is an extensive literature addressing the physical processes that govern the rate-dependent softening response within shear bands, notably Vardoulakis $(2000,2002)$ and more recent work such as Cecinato et al. (2011) and Veveakis et al. (2013). However, the present paper is concerned with the net effect of a softening and propagating shear band, not the detailed physics, for which a simple phenomenological model of the shear band response is sufficient.

A particular form of shear band propagation, which has received considerable attention recently (Quinn et al., 2011, 2012; Locat et al., 2011, 2013), is a progressive spreading failure accompanied by an uphill growth of the shear band driven by the removal of the downslope support. In contrast, catastrophic failure can occur by spontaneous growth of an initial shear band at both ends under existing forces (Puzrin et al., 2004; Puzrin \& Germanovich, 2005; Andresen \& Jostad, 2007; Viesca et al., 2008; Garagash \& Germanovich, 2012; Viesca \& Rice, 2012; Viesca, personal communication, 2014; Puzrin et al., 2015). Analysis of the latter form of failure requires an assumption with respect to the 'history' leading to the initiation of the shear band propagation in a catastrophic landslide. In the work reference above, one particular type of the initiation history has been assumed: the initial shear band grows progressively through the homogeneous intact material, until its length reaches a critical value and further propagation becomes catastrophic. Under

Manuscript received 27 October 2014; revised manuscript accepted 23 April 2015. Published online ahead of print 27 July 2015.

Discussion on this paper closes on 1 February 2016, for further details see p. ii.

* Centre for Offshore Foundation Systems, The University of Western Australia, Crawley, WA, Australia.

$\dagger$ Institute for Geotechnical Engineering, ETH Zurich, Zurich, Switzerland. such conditions, prior to catastrophic failure, the material is fully softened not only within the shear band, but also in the process zone adjacent to the shear band.

In some cases, however, a different type of shear band initiation history can be more relevant: when the initial fully softened shear band forms either instantaneously or progressively within a pre-existing weak zone. Such phenomena occur in nature and are attributable to, for example, local increase in the excess pore water pressures in a weaker soil lens parallel to the slope due to seismic shaking, gas hydrate disassociation, and so on. If the length of this weak zone is larger than some critical value, the shear band will propagate catastrophically outside the weak zone into the enclosing material. In such a case, prior to the catastrophic failure, the fully softened shear band and its process zone are located in two different materials, and the process zone may not reach a full softened state even at its boundary with the shear band; that is, the catastrophic failure can start earlier than in the homogeneous material.

This paper seeks to explore the latter initiation scenario and its effects on the catastrophic failure by deriving simple analytical criteria for the shear band propagation from the initial weak zone into the enclosing material of the infinite slope. Analytical criteria (e.g. Puzrin \& Germanovich, 2005; Puzrin et al., 2015) have a limitation of being based on simplifying assumptions; this is, however, greatly outweighed by the convenience of their application to geographic information system (GIS) based deterministic and probabilistic stability analysis of large-scale offshore developments (Rushton et al., 2015), provided they can be validated using numerical methods and palaeo-landslide data (Gray et al., 2015).

Focusing on cases where the length of the process zone is not negligible relative to that of the initial failure zone, this paper extends the existing stability criteria (Puzrin \& Germanovich, 2005) to account for: (a) the weak zone initiation scenario; $(b)$ different shear strength parameters in the initial failure zone and in the process zone; $(c)$ more general forms of softening response rather than just the linear degradation relationship that has been considered to date; and $(d)$ elastic deformations in the shear band and in the entire sliding layer. The obtained criteria and underlying assumptions are validated against the numerical results. 
To this end, a simplified geometry of the form shown in Fig. 1 is adopted. In the work of Puzrin \& Germanovich (2005) and Viesca (personal communication, 2014), who provided approximate and exact propagation criteria, respectively, the length, $l$, of shear band was defined to include partially softened process zones of lengths $\omega_{1}$ and $\omega_{2}$ at either end of a fully softened initial failure zone, implying that all the zones are formed of the same material governed by the same shear strength degradation relationship. In contrast, here the analysis considers a fully 'pre-softened' initial failure zone of length, $l_{0}$, formed in a thin lens of a weaker material, flanked by a stronger material, where the softening has not yet been completed, but displacements are large enough for the initial failure zone to reach residual strength along its entire length. The slide is then brought to failure by gradually increasing the gravity loading from the overlying layer of thickness, $h$, resulting in the development of partially softened process zones at either end of the initial failure zone.

Within the shear band shown in Fig. 1(b), the material response is as shown schematically in Fig. 2, where $\gamma$ and $\delta$ are the shear strain and the relative displacement, respectively, across the shear band, with superscripts 'e' and 'p' used to denote elastic and plastic components. Puzrin \& Germanovich (2005) ignored any elastic component, but this is included in the present study. Also the shear band is often considered as vanishingly thin, so the softening curve from peak strength, $\tau_{\mathrm{p}}$, to residual strength, $\tau_{\mathrm{p}}$ occurs over a distance, $\delta_{\mathrm{r}}^{\mathrm{p}}$. Here it is necessary to consider a finite width, $s$, of the shear band, with the softening related to plastic strain and residual strength reached after plastic strain, $\gamma_{\mathrm{r}}^{\mathrm{p}}=\delta_{\mathrm{r}}^{\mathrm{p}} / s$. In order to distinguish between the shear strength parameters inside the initial failure zone $l_{0}$, and within the process zones, the former are denoted as $\tau_{\mathrm{p} 0}, \tau_{\mathrm{r} 0}$ and $\delta_{\mathrm{r} 0}^{\mathrm{p}}$. Both linear and exponential softening curves are considered, although initial focus is on the former.

\section{Background theory}

Although the failure mechanism of catastrophic landslides has been widely discussed (Puzrin et al., 2004; Viesca \& Rice, 2012), robust criteria that allow quantitative prediction of the conditions for catastrophic failure have yet to be developed for general cases involving finite lengths of the process zones. Palmer \& Rice (1973) originally applied linear elastic fracture mechanics and the J-integral (Rice, 1968) to analyse the progressive failure for an excavated cut slope of overconsolidated London clay. This analytical method, designated as

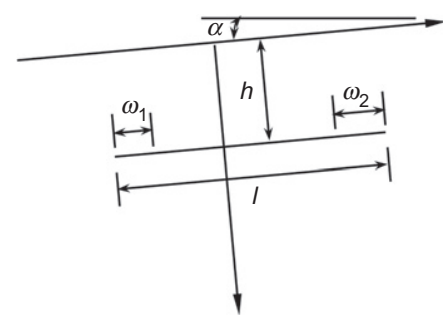

(a)

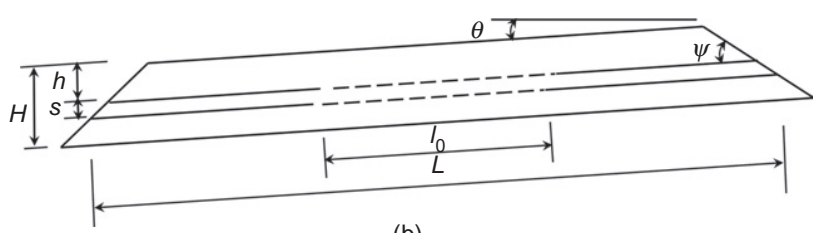

(b)

Fig. 1. Conceptual model used (a) by Puzrin \& Germanovich (2005), and (b) in this study

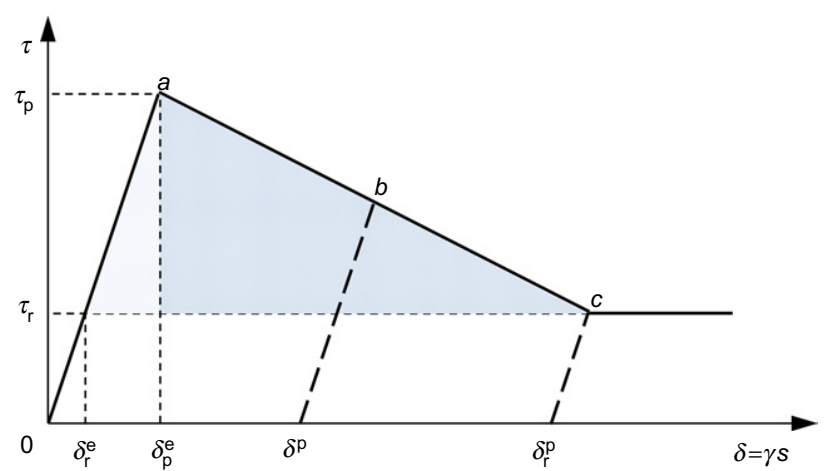

(a)

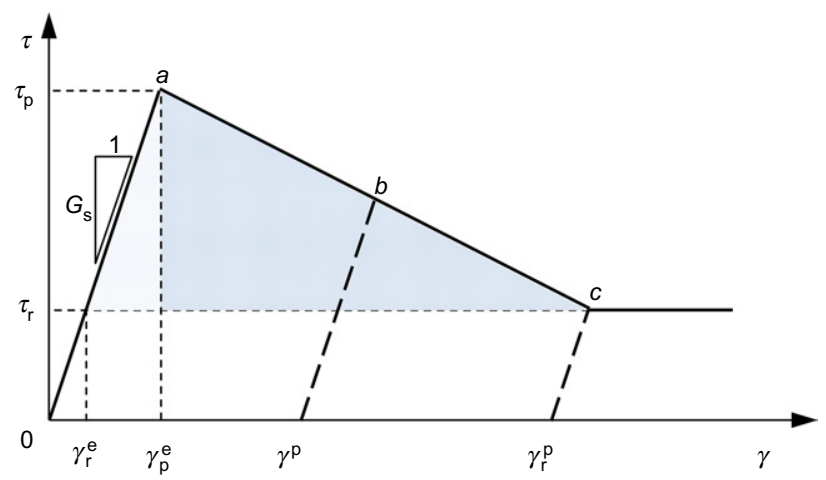

(b)

Fig. 2. Linear degradation of shear strength with plastic displacement in shear band: (a) shear stress-shear displacement; (b) shear stressshear strain

PR, was applied by Quinn et al. (2011, 2012) to study progressive (retrogressive) failure of a Canadian sensitive clay slope with an excavated cut.

The PR method was extended by Puzrin \& Germanovich (2005) to apply to sands and soft (normally consolidated or lightly overconsolidated) clays, with different elastic moduli in loading and unloading, in which an ultra-thin shear band parallel to an infinite slope surface was assumed, as shown in Fig. 1(a). At each tip of the shear band, there is a process zone (lengths $\omega_{1}$ and $\omega_{2}$ ) within which the shear strength reduces from peak (at the extremity of the shear band) to residual (at the transition to the central fully softened zone formed in the same material as the process zones). For a particular case of identical elastic moduli in loading and unloading and for conditions where the length of each process zone is negligible by comparison with the length $l$ of the shear band, their stability criterion reduces to what will be referred to here as $\mathrm{PG}_{1}$, whereby the shear band in the infinite slope will propagate if its length $l$ exceeds a critical value, given by

$$
l \geq 2 \frac{l_{u}}{r}
$$

where

$$
r=\frac{\tau_{\mathrm{g}}-\tau_{\mathrm{r}}}{\tau_{\mathrm{p}}-\tau_{\mathrm{r}}}
$$

is the gravitational shear stress ratio, with $\tau_{\mathrm{p}}, \tau_{\mathrm{r}}$ and $\tau_{\mathrm{g}}$ representing the peak, residual and gravity shear stress, respectively. The characteristic length, $l_{u}$, is given by

$$
l_{u}=\sqrt{\frac{2 E^{\prime} \bar{\delta} h}{\tau_{\mathrm{p}}-\tau_{\mathrm{r}}}}
$$


where $\bar{\delta}$ is the characteristic displacement in the process zone, and $E^{\prime}$ is the plane strain modulus of the elastic material above the shear band. In turn the characteristic displacement is derived as

$$
\bar{\delta}=\frac{\int\left(\tau-\tau_{\mathrm{r}}\right) \mathrm{d} \delta^{\mathrm{p}}}{\tau_{\mathrm{p}}-\tau_{\mathrm{r}}}
$$

where $\tau$ is the shear stress in the process zone.

As noted above, this criterion assumes that the process zones, $\omega$ (where $\omega_{1}=\omega_{2}=\omega$ for an infinite slope), occupy an insignificant fraction of the shear band (i.e. $2 \omega / l<<1$ ) and thus the shear strength within the shear band is uniform at the residual value. This gives an upper bound of driving force of $\left(\tau_{\mathrm{g}}-\tau_{\mathrm{r}}\right) l$. Puzrin \& Germanovich (2005) further proposed an approximate criterion (termed ' $\mathrm{PG}_{2}$ ' hereafter) for conditions where the process zones are a significant proportion of the shear band, which may be expressed as

$$
l \geq\left(1+\frac{r \pi}{2}-r\right) 2 \frac{l_{u}}{r}
$$

Given its simplicity and conservatism relative to $\mathrm{PG}_{2}$, the $\mathrm{PG}_{1}$ criterion has been applied to a number of practical applications to predict landslides (Puzrin et al., 2004; Puzrin \& Germanovich, 2005). While in many real-life cases the length of the process zone is significant, defining the catastrophic failure criteria in terms of the overall length of the shear band is not very practical since the length of the process zone is difficult to determine a priori. In contrast, the length of the initial failure zone is easier to predict, in particular if it forms within a lens of a weaker, fully softened material, but the existing criteria are not capable of dealing with different materials of the initial failure zone and the process zone.

\section{Outline of present study}

In the present study, growth of an initial pre-softened shear failure zone under undrained conditions is investigated analytically and numerically. The work targets the role of the process zones, with the aim of developing propagation criteria for catastrophic failure that take account of elastic deformations in the shear band and in the entire sliding layer. Analytical solutions are presented for both a linear strength degradation curve and an exponential degradation curve suggested by Einav \& Randolph (2005). The resulting stability criteria are developed for a general case of a different material in the initial failure zone and compared with those from Puzrin \& Germanovich (2005).

For the numerical analysis, a modified Tresca model considering strain-softening behaviour of the soil was incorporated into numerical simulations using a static largedeformation finite-element (LDFE) approach. Conventional finite-element methods based on a Lagrangian mesh can experience severe mesh distortion caused by development of the shear band, while mesh distortion is avoided in the LDFE analyses. Numerical results are presented, and compared with the analytical solutions, focusing primarily on the linear strength degradation curve, but also showing summary results for the exponential degradation.

\section{THEORETICAL PROPAGATION CRITERIA}

To simplify the problem, the authors have assumed the plane strain model is symmetric (up and down the slope) and focused on the left half, as shown in Fig. 3. If there was no discontinuity, the slope down to the depth $h$ would be subjected to an initial internal lateral force per unit thickness $\left(P_{\mathrm{g}}\right)$ caused by earth pressure, and an initial shear stress $\left(\tau_{\mathrm{g}}\right)$ caused by the soil weight and slope angle. Note, all 'forces' referred to in the following are forces per unit (out of plane) thickness. Conceptually, the problem is explored by fixing a length, $l_{0}$, of initial failure zone, formed in a thin lens of a weaker material where the strength has been reduced to its residual value, $\tau_{\mathrm{r} 0}$, along its entire length, flanked by stronger material where the softening has not yet been completed. Then the slope is brought to catastrophic failure by increasing the gravity loading such as might occur due to sedimentation or increase in slope angle caused by diapirism.

The initial failure zone within the slope will disturb the stress and strain fields in its vicinity for any level of gravity loading, $\tau_{\mathrm{g}}$, that exceeds the residual shear strength, $\tau_{\mathrm{r} 0}$, in this zone. As the relative magnitude of $\tau_{\mathrm{g}}$ increases (relative to the peak and residual strengths outside the initial failure zone, $\tau_{\mathrm{p}}$ and $\tau_{\mathrm{r}}$ ), first a region of elastic shear and then the process zone will start to grow adjacent to the initial failure zone (Bernander, 2000, 2008; Locat et al., 2011), with plastic deformations causing partial softening. As it does so, the internal lateral force $P$ will increase above $P_{\mathrm{g}}$ within the elastic and process zones and the leading half of the pre-softened zone. In general, the $x$ coordinate at the end of the process zone (start of the pre-softened zone) is taken as $x_{1}$, which approaches a value $\omega$ at the critical condition of catastrophic failure.

Figure 4 shows profiles of key variables (shear stress $\tau, P, \delta^{\mathrm{e}}$ and $\delta^{\mathrm{p}}$ ) at three stages of gradually increasing gravity shear stress, $\tau_{\mathrm{g}}:(a)$ where the peak strength had just been mobilised due to elastic deformations in the shear band immediately adjacent to the pre-softened zone (stage 1); $(b)$ at the onset of catastrophic failure, where the lateral force, $P$, in the material above the shear band has reached a maximum value, $P_{\max }$, at the interface between the pre-softened and process zones (stage 2); and (c) a stage just after catastrophic failure where additional displacement has caused the shear strength of the shear band in the process zone to degrade below $\tau_{\mathrm{g}}$ and towards the residual value at the interface with the presoftened zone (stage 3 ). For convenience with later algebra, the origin, $\mathrm{O}$, of the Cartesian coordinates system in Fig. 3 has been placed at the interface between the process zone and the region of purely elastic deformations, and thus moves as the gravity shear stress increases.

To the left of the axis origin $(x \leq 0)$, the shear band deforms elastically with $\tau_{\mathrm{g}} \leq \tau \leq \tau_{\mathrm{p}}$, while for $x>0$ plastic conditions apply and the strength degrades from peak (at $x=0$ ), in general with a step change to $\tau_{\mathrm{r} 0}$ at the start of the pre-softened zone. Note that this singularity in shear strength and mobilised shear stress would in reality occur over a finite distance, but short within the overall geometry of the problem, hence idealised as a step change. As will be proved analytically later, the critical condition occurs when the

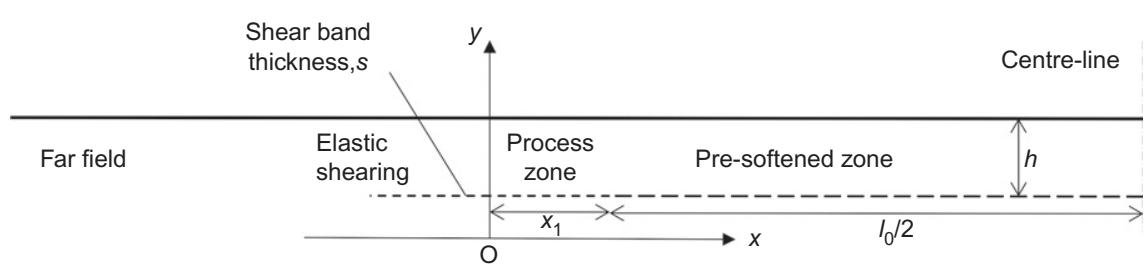

Fig. 3. Coordinate system and identification of different zones 
gravity loading has increased to a level where the shear strength adjacent to the pre-softened zone has just been reduced to the gravity shear stress, $\tau_{\mathrm{g}}$. For that condition, the lateral force in the process zone is a maximum at $x=x_{1}$ (since the gradient of $P$ is proportional to $\tau-\tau_{\mathrm{g}}$ ).

Additional displacement beyond the critical condition will lead to further degradation of the shear strength in the process zone at $x=x_{1}$. Stability can then no longer be maintained, since the location of the maximum force moves to within the process zone so that the force at the interface between the process and pre-softened zones reduces. This is incompatible with the required increase in driving force from the pre-softened zone, as illustrated in Fig. 4(b) by the discontinuity in force $P$ at the interface with the process zone. Essentially, if the driving force derived from the pre-softened zone becomes larger than $P_{\max }$, the process zone develops further but the resisting force at the interface with the pre-softened zone must reduce below $P_{\max }-$ resulting in instability. Stage 2 in Fig. 4, where there is still a strength discontinuity between the process and pre-softened zones, represents the critical condition for catastrophic failure.

The analysis that follows, which extends that in Puzrin \& Germanovich (2005) to include elastic deformations within the shear band and in the entire sliding layer, considers 'net' values of variables with respect to those that would exist in an infinite slope without any pre-softened zone. Hence $\Delta \delta^{\mathrm{p}}=\delta^{\mathrm{p}}$, $\Delta \delta^{\mathrm{e}}=\delta^{\mathrm{e}}-\delta_{\mathrm{g}}^{\mathrm{e}}, \Delta \tau=\tau-\tau_{\mathrm{g}}$ and $\Delta P=P-P_{\mathrm{g}}$. This convention gives $\Delta \tau_{\mathrm{p}}=\tau_{\mathrm{p}}-\tau_{\mathrm{g}}=(1-r)\left(\tau_{\mathrm{p}}-\tau_{\mathrm{r}}\right), \Delta \tau_{\mathrm{r}}=\tau_{\mathrm{r}}-\tau_{\mathrm{g}}=-r\left(\tau_{\mathrm{p}}-\tau_{\mathrm{r}}\right)$ and

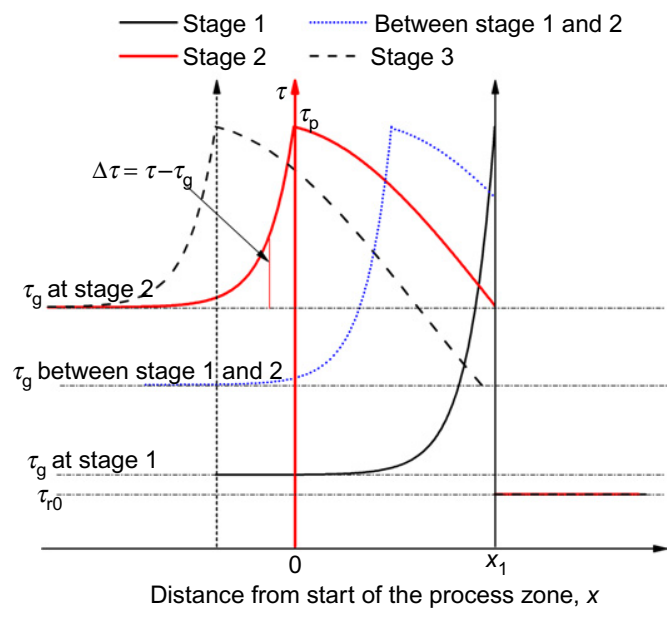

(a)

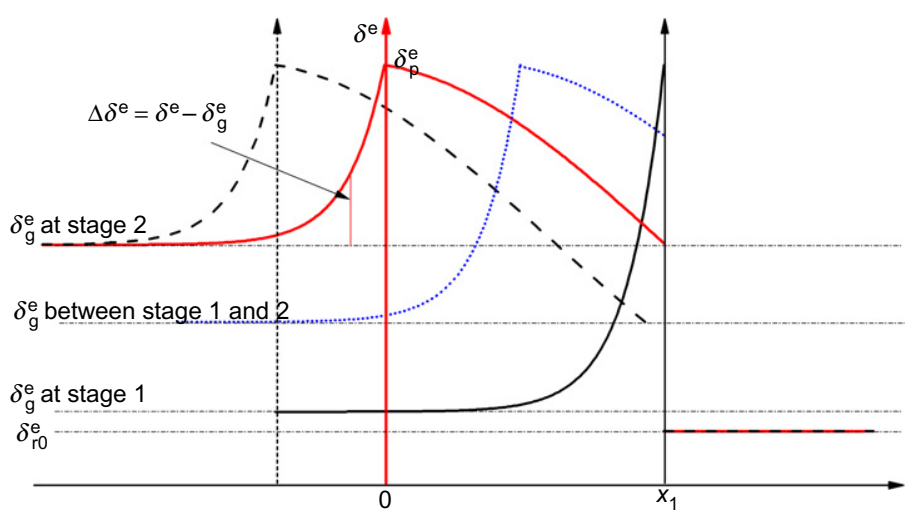

Distance from start of the process zone, $x$

(c)

$$
\begin{aligned}
\Delta \tau_{\mathrm{r} 0} & =\tau_{\mathrm{r} 0}-\tau_{\mathrm{g}}=-r_{0}\left(\tau_{\mathrm{p}}-\tau_{\mathrm{r}}\right), \text { where } \\
r_{0} & =\frac{\tau_{\mathrm{g}}-\tau_{\mathrm{r} 0}}{\tau_{\mathrm{p}}-\tau_{\mathrm{r}}}
\end{aligned}
$$

Figure 5 presents the equilibrium of the sliding layer (depth $h$ ), which is given by

$$
\frac{\mathrm{d} \Delta P}{\mathrm{~d} x}=\Delta \tau
$$

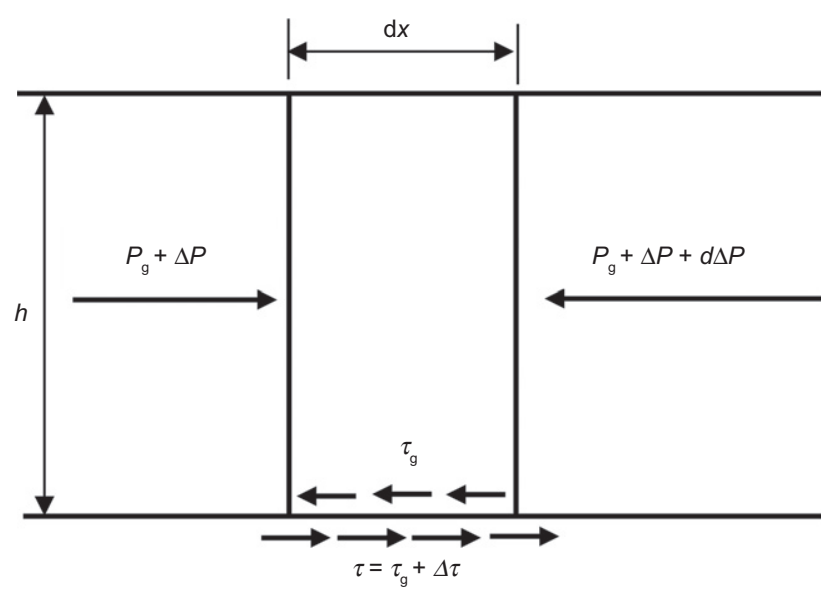

Fig. 5. Equilibrium of the sliding layer

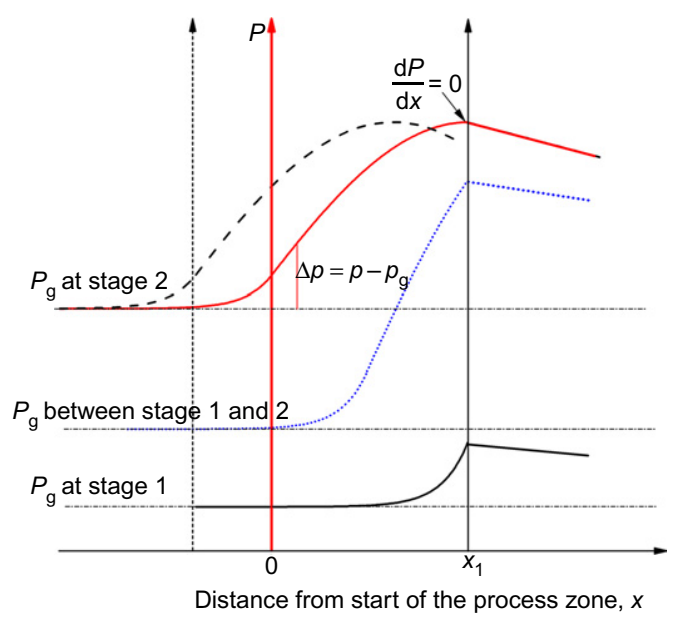

(b)

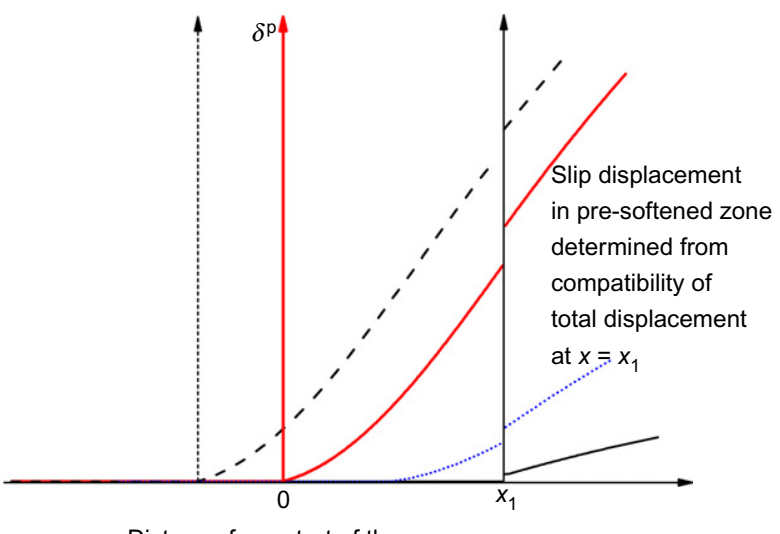

Distance from start of the process zone, $x$

(d)

Fig. 4. Profiles of key variables at different stages of failure: (a) shear stress; (b) lateral force; (c) elastic shear displacement within shear band; (d) plastic shear displacement within shear band 
In addition, compatibility must be satisfied between the net displacements across the shear band and displacements, $\Delta u$, within the overlying sliding layer (Palmer \& Rice, 1973; Puzrin et al., 2004; Puzrin \& Germanovich, 2005). This assumption suits a base with much higher stiffness than the weak layer, that is, where the base displacements can be neglected. Since the compressional strain $\mathrm{d}(\Delta u) / \mathrm{d} x$ within the sliding layer is equal to $\Delta P / E^{\prime} h$, and taking $\Delta \delta=\Delta u$, it is possible to write

$$
\frac{\mathrm{d} \Delta \delta}{\mathrm{d} x}=\frac{\Delta P}{E^{\prime} h}
$$

where

$$
\Delta \delta=\Delta \delta^{\mathrm{e}}+\Delta \delta^{\mathrm{p}}=\frac{\Delta \tau}{G_{\mathrm{s}}} s+\Delta \delta^{\mathrm{p}}
$$

In these relationships, $E^{\prime}$ is the (average) plane strain modulus of the sliding layer of thickness $h$, and $G_{\mathrm{s}}$ is the shear modulus of the soil in the shear band (thickness, $s$ ). Note that it is sufficient to consider the net change in force, $\Delta P$, in equation (8) rather than the total force, since for an infinite slope the displacement due to the geostatic earth pressure, $P_{\mathrm{g}}$, will be uniform at $\delta_{\mathrm{g}}^{\mathrm{e}}$ (prior to creation of the pre-softened shear band).

Expressions for the key variables are derived below, within the region of purely elastic shearing and process zones, respectively. These result in a new propagation criterion for catastrophic failure with respect to the length of the original pre-softened shear band.

\section{Elastic shearing}

In the elastic regime (for $x \leq 0), \Delta \delta^{\mathrm{p}}=0$ and thus combining equations (7) to (9) leads to

$$
\frac{\mathrm{d} \Delta \tau}{\mathrm{d} x}=\frac{G_{\mathrm{s}}}{s} \frac{\mathrm{d} \Delta \delta^{\mathrm{e}}}{\mathrm{d} x} \Rightarrow \frac{\mathrm{d}^{2} \Delta P}{\mathrm{~d} x^{2}}=\frac{G_{\mathrm{s}}}{E^{\prime} h s} \Delta P
$$

The solution of the governing equation in $\Delta P$, subject to boundary conditions of $\Delta P$ tending to zero at large negative $x$, and $\Delta \tau=\Delta \tau_{\mathrm{p}}$ at $x=0$, is

$$
\Delta P=\Delta \tau_{\mathrm{p}} l_{u, \mathrm{e}} \mathrm{e}^{x / l_{u, \mathrm{e}}}
$$

and

$$
\Delta \tau=\Delta \tau_{\mathrm{p}} \mathrm{e}^{x / l_{u, \mathrm{e}}} ; \quad \Delta \delta^{\mathrm{e}}=\Delta \delta_{\mathrm{p}}^{\mathrm{e}} \mathrm{e}^{x / l_{u, \mathrm{e}}}
$$

where, in the spirit of Puzrin \& Germanovich (2005), a characteristic length, $l_{u, \mathrm{e}}$, is introduced for purely elastic shearing, given by

$$
l_{u, \mathrm{e}}=\sqrt{\frac{E^{\prime} h s}{G_{\mathrm{s}}}}
$$

The exponential term provides a shape factor for $\Delta P, \Delta \tau$ and $\Delta \delta^{\mathrm{e}}$, and it may be noted in passing that $\Delta P=\Delta \tau l_{u, \mathrm{e}}$ in the elastic zone.

For conditions shown as stage 1 in Fig. 4, where the shear stress has just reached $\tau_{\mathrm{p}}$ at the interface with the pre-softened zone, the driving force from that zone is

$$
\left.\Delta P\right|_{x=0}=-\Delta \tau_{\mathrm{r} 0} \frac{l_{0}}{2}=r_{0}\left(\tau_{\mathrm{p}}-\tau_{\mathrm{r}}\right) \frac{l_{0}}{2}
$$

Equating this to the value of $\Delta P$ emerging from the elastic solution leads to a relationship between the length of the pre-softened zone and the normalised shear stress level, $r_{\mathrm{e}}$, at this 'elastic limit', which is given by

$$
l_{0}=\left(1-r_{\mathrm{e}}\right) \frac{2 l_{u, \mathrm{e}}}{r_{0, \mathrm{e}}}
$$

where $r_{0, \mathrm{e}}$ is the value of $r_{0}$ for this stage. This relationship echoes those of equations (1) and (5) quoted from Puzrin \& Germanovich (2005).

\section{Process zone - linear degradation}

Within the process zone $\left(0<x<x_{1}\right)$, the shear stress in the shear band is limited to the current shear strength, which, for the linear strain-softening behaviour, is given by

$$
\tau=\tau_{\mathrm{p}}+\left(\tau_{\mathrm{r}}-\tau_{\mathrm{p}}\right) \frac{\delta^{\mathrm{p}}}{\delta_{\mathrm{r}}^{\mathrm{p}}}
$$

where $\delta_{\mathrm{r}}^{\mathrm{p}}$ is the value of $\delta^{\mathrm{p}}$ required to soften to the residual strength, as shown in Fig. 2(a). Thus the net value $\Delta \tau$ is given by

$$
\Delta \tau=\left(\tau_{\mathrm{p}}-\tau_{\mathrm{r}}\right)\left(1-r-\frac{\delta^{\mathrm{p}}}{\delta_{\mathrm{r}}^{\mathrm{p}}}\right)
$$

According to equation (4), $\bar{\delta}$ may be calculated as $\delta_{\mathrm{r}}^{\mathrm{p}} / 2$, hence the characteristic length $l_{u}$ is given by

$$
l_{u}=\sqrt{\frac{2 E^{\prime} \bar{\delta} h}{\tau_{\mathrm{p}}-\tau_{\mathrm{r}}}}=\sqrt{\frac{E^{\prime} h \delta_{\mathrm{r}}^{\mathrm{p}}}{\tau_{\mathrm{p}}-\tau_{\mathrm{r}}}}
$$

By substituting equation (17), equations (7) and (8) may be re-rewritten as

$$
\begin{aligned}
& \frac{\mathrm{d} \Delta \hat{P}}{\mathrm{~d} \hat{x}}=\frac{\Delta \tau}{\left(\tau_{\mathrm{p}}-\tau_{\mathrm{r}}\right)}=1-r-\hat{\delta}^{\mathrm{p}} \\
& \Delta \hat{P}=\beta^{2} \frac{\mathrm{d} \hat{\delta}^{\mathrm{p}}}{\mathrm{d} \hat{x}}
\end{aligned}
$$

where

$$
\beta^{2}=1-\frac{\left(\tau_{\mathrm{p}}-\tau_{\mathrm{r}}\right) s}{G_{\mathrm{s}} \delta_{\mathrm{r}}^{\mathrm{p}}}=1-\left(\frac{l_{u, \mathrm{e}}}{l_{u}}\right)^{2}
$$

and normalised variables have been introduced, comprising $\Delta \hat{P}=\Delta P / l_{u}\left(\tau_{\mathrm{p}}-\tau_{\mathrm{r}}\right), \hat{x}=x / l_{u}$ and $\hat{\delta}^{\mathrm{p}}=\delta^{\mathrm{p}} / \delta_{\mathrm{r}}^{\mathrm{p}}$. Note that introduction of elasticity in the shear band results in $\beta<1$ ( $\beta$ is always positive in the current study), by contrast with the corresponding $\mathrm{PG}_{2}$ solution in Puzrin \& Germanovich (2005), where $\beta=1$.

Combining equations (19) and (20) yields

$$
\frac{\mathrm{d}^{2} \hat{\delta}^{\mathrm{p}}}{\mathrm{d} \hat{x}^{2}}=\frac{1-r-\hat{\delta}^{\mathrm{p}}}{\beta^{2}}
$$

The boundary conditions for $\hat{\delta}^{p}$ are obtained at $\hat{x}=0$ where, in order to match the conditions in the region of elastic shear, which are

$$
\begin{aligned}
& \left.\hat{\delta}^{\mathrm{p}}\right|_{\hat{x}=0}=0 ; \\
& \left.\quad \frac{\mathrm{d} \hat{\delta}^{\mathrm{p}}}{\mathrm{d} \hat{x}}\right|_{\hat{x}=0}=\left.\frac{1}{\beta^{2}} \Delta \hat{P}\right|_{\hat{x}=0}=(1-r) \frac{\sqrt{1-\beta^{2}}}{\beta^{2}}
\end{aligned}
$$

where the second boundary condition makes use of equations (11) and (20), substituting for $l_{u, \mathrm{e}}$ from equation (21). Solving equation (22) with these boundary conditions gives

$$
\hat{\delta}^{\mathrm{p}}=(1-r)\left[1-\frac{1}{\beta} \sin \left(\frac{\hat{\omega}-\hat{x}}{\beta}\right)\right]
$$


where a normalised length $\hat{\omega}=\omega / l_{u}$ has been introduced, given by

$$
\hat{\omega}=\beta \arcsin (\beta)
$$

Substituting equation (24) into equation (20) yields a corresponding solution for the force

$$
\Delta \hat{P}=(1-r) \cos \left(\frac{\hat{\omega}-\hat{x}}{\beta}\right)
$$

From equation (19), the normalised shear strength in the process zone may be obtained as

$$
R(\hat{x})=\frac{\Delta \tau}{\Delta \tau_{\mathrm{p}}}=\frac{1}{\beta} \sin \left(\frac{\hat{\omega}-\hat{x}}{\beta}\right)
$$

The solution shows that the maximum force in the process zone is reached at $\hat{x}=\hat{\omega}$ where both $\Delta \hat{P}$ and $\hat{\delta}^{p}$ are equal to $1-r$ and $\Delta \tau$ is zero. These correspond to the conditions in Fig. 4 at the end of stage 2, at the point of catastrophic failure. Hence the critical length of the process zone is $x_{1}=\omega$. This also provides a condition justifying the assumption of the fully softened initial failure zone at the moment of catastrophic failure

$$
\delta_{\mathrm{r} 0}^{\mathrm{p}} \leq(1-r) \delta_{\mathrm{r}}^{\mathrm{p}}
$$

The critical value of $l_{0}$ for catastrophic failure may be obtained by noting that the normalised force accumulated in the pre-softened zone is

$$
\left.\Delta \hat{P}\right|_{\hat{x}=\hat{\omega}}=\frac{-\Delta \tau_{\mathrm{r} 0} l_{0}}{2 l_{u}\left(\tau_{\mathrm{p}}-\tau_{\mathrm{r}}\right)}=\frac{r_{0} \hat{l}_{0}}{2}
$$

where $\hat{l}_{0}=l_{0} / l_{u}$. Satisfying equilibrium at $\hat{x}=\hat{\omega}$ results in a criterion for catastrophic failure in terms of the initial length of failure zone

$$
l_{0}=(1-r) \frac{2 l_{u}}{r_{0}}
$$

It is also of interest to evaluate the total critical length of the pre-softened and process zones, to compare with the $\mathrm{PG}_{2}$ estimate of the critical length in equation (5). The critical total length, $l$, is derived as

$$
l=l_{0}+2 \omega=\left[(1-r)+r_{0} \beta \arcsin (\beta)\right] \frac{2 l_{u}}{r_{0}}
$$

In order to compare equation (31) with the $\mathrm{PG}_{2}$ criterion, it is important to note that the latter cannot account for different materials of the initial failure zone and the process zone. Therefore, for this and other comparisons with existing criteria and with numerical simulations it has been assumed that the residual strength values in the initial failure zone and the process zones are identical, that is, $r_{0}=r$. This, however, does not contradict the assumption of the fully softened initial failure zone, provided condition (28) is satisfied. In this case, for $\beta=1$ equation (31) becomes identical to the $\mathrm{PG}_{2}$ criterion, even though in the $\mathrm{PG}_{2}$ solution the strength at the end of the process zone $(x=\omega)$ was assumed to be fully remoulded, that is, $\left.\hat{\delta}^{\mathrm{p}}\right|_{\hat{x}=\hat{\omega}}=1$ rather than $1-r$.

In a natural slope, where an initial fully softened zone forms within a lens of a weaker material, the length of the process zone is very hard, if not completely impossible, to measure. Therefore, the $l_{0}$ based propagation criterion, that is, equation (30), is considered more useful compared with equation (31). In order to avoid initiation of a process zone with plastic deformations, the length of pre-softened zone should satisfy equation (15).

In summary, three stages of progressive failure of a clay slope with a softenable shear band may be distinguished: the first is purely elastic shearing, $l_{0} \leq(1-r)\left(2 l_{u, \mathrm{e}} / r_{0}\right)$; the second is development of the process zone, $(1-r)$ $\left(2 l_{u, \mathrm{e}} / r_{0}\right)<l_{0}<(1-r)\left(2 l_{u} / r_{0}\right)$; and the third is catastrophic failure $l_{0} \geq(1-r)\left(2 l_{u} / r_{0}\right)$. These three stages are identified schematically in the $l_{0} / l_{u}-1 / r$ plane for the case of $r_{0}=r$, as shown in Fig. 6, which may be used to estimate the state of a clay slope with respect to catastrophic failure. For comparison, the original $\mathrm{PG}_{1}$ solution (equation (1)) is also plotted, suggesting that for the shear band initiation scenario considered here, where the initial shear band is formed within the fully softened zone of a weaker material, this criterion cannot provide a conservative estimate with respect to the length of the fully softened zone, although for practical values of $\beta(>0.9)$ it remains conservative in respect of the total length of fully softened and process zones for the linear strain-softening relationship.

The expression of the process zone length, that is, equation (25) is now revisited. It is related to the value of parameter $\beta$; this is a function of $l_{u, \mathrm{e}} / l_{u}$, which in turn reflects the ratio of elastic and plastic shear strains between peak and residual shear strength. If $l_{u, \mathrm{e}} / l_{u} \rightarrow 0$ (or $\beta \rightarrow 1$ ) the effect of elastic deformations in the shear band is slight and the length of the process zone is considerable, while if $l_{u, \mathrm{e}} / l_{u} \rightarrow 1$ (or $\beta \rightarrow 0$ ) the relative length of the process zone reduces to zero and elastic deformations dominate. The former scenario corresponds to the $\mathrm{PG}_{2}$ assumption and in this case equation (31) is identical to the $\mathrm{PG}_{2}$ solution. For the somewhat hypothetical case of $l_{u, \mathrm{e}} / l_{u}>1$ (mathematically $\beta^{2}<0$ ), the solution presented, equation (30), is no longer valid. However, the critical condition would be reached once elastic shear deformations were fully mobilised (i.e. the propagation criterion becomes equation (15), with catastrophic brittle failure occurring as a result of the elastic unloading stiffness being less than the rate of plastic degradation).

It is noted that for elastic shearing, the accurate expression for $\Delta P$ is

$$
\Delta P=\int_{0}^{h} E^{\prime} \frac{\mathrm{d} \Delta u}{\mathrm{~d} x} \mathrm{~d} y
$$

Since $\Delta u$ is the horizontal displacement within the overlying material (relative to conditions where $\tau=\tau_{\mathrm{g}}$ at the base of the layer), $\mathrm{d} \Delta u / \mathrm{d} x$ is a function of depth $y$. Equation (8) is obtained by assuming that the average value of $\mathrm{d} \Delta \delta^{\mathrm{e}} / \mathrm{d} x$ through the depth of overlying soil is identical to the value in the shear band. This assumption will cause a degree of error in the analysis of the elastic zone, but will be balanced by similar error in calculating

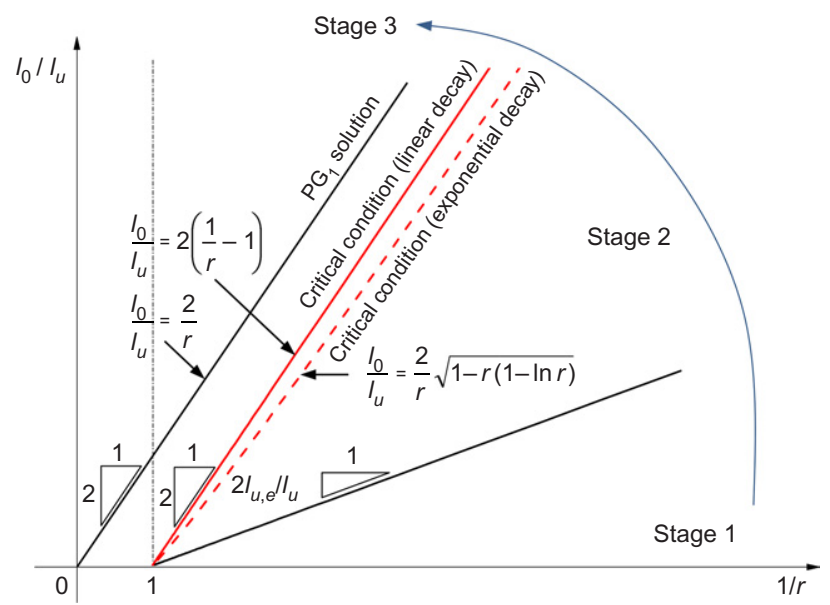

Fig. 6. Distinguishing features of three stages in $l_{0} / l_{u}-1 / r$ plane $\left(r_{0}=r\right)$ : stage 1: development of the elastic shearing; stage 2: development of the process zone; stage 3: catastrophic failure 
the elastic component of displacements in the process zone. These compensating errors are discussed further in light of the results from numerical analysis of the problem presented later, but are expected to have little effect on the final critical condition for catastrophic failure shown in Fig. 6.

Before turning to the numerical analysis, the effect on the critical conditions of different strain-softening relationships within the process zone will be investigated by considering an exponential degradation of shear strength with plastic shear strain.

\section{Process zone - exponential degradation}

The strength degradation relationship suggested by Einav \& Randolph (2005) was adopted as an alternative to linear degradation, with the shear strength reducing from peak to residual as an exponential function of the cumulative plastic shear strain, $\gamma^{\mathrm{p}}$ (see Fig. 7(b)). They introduced a normalising parameter, $\gamma_{95}^{\mathrm{p}}$, representing the plastic shear strain to achieve $95 \%$ reduction in shear strength. Substituting the displacement across the shear band, $\delta=\gamma s$, the net value of limiting shear stress may be derived as

$$
\Delta \tau=\tau-\tau_{\mathrm{g}}=\left(\tau_{\mathrm{p}}-\tau_{\mathrm{r}}\right)\left(-r+\mathrm{e}^{-3 \delta^{\mathrm{p}} / \delta_{95}^{\mathrm{p}}}\right)
$$

where $\delta_{95}^{\mathrm{p}}$ is the value of $\delta^{\mathrm{p}}$ (for exponential degradation) at which the material has experienced $95 \%$ of the reduction in strength due to softening. The characteristic displacement $\bar{\delta}$ in the process zone can be expressed as

$$
\bar{\delta}=\frac{\int\left(\tau-\tau_{r}\right) \mathrm{d} \delta}{\tau_{\mathrm{p}}-\tau_{\mathrm{r}}}=\frac{1}{3} \delta_{95}^{\mathrm{p}}
$$

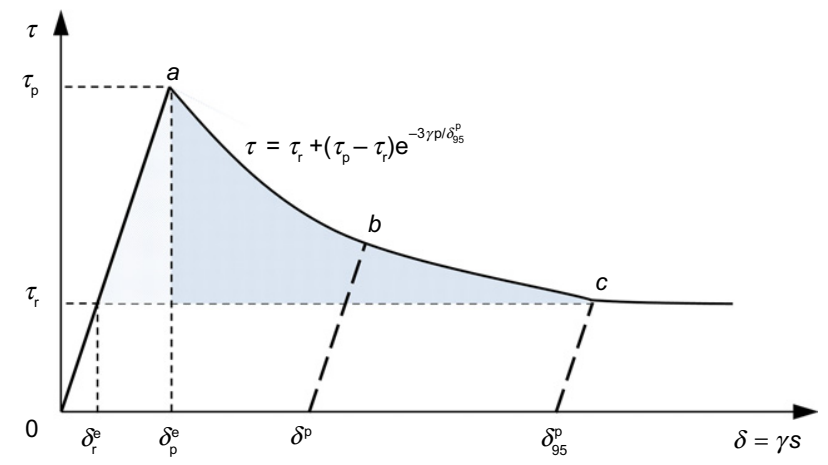

(a)

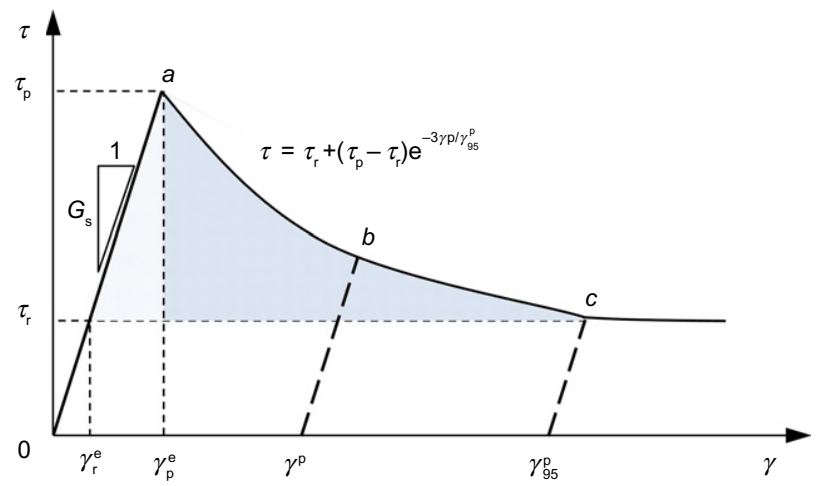

(b)

Fig. 7. Exponential degradation of shear strength with plastic displacement in shear band: (a) shear stress-shear displacement; (b) shear stress-shear strain
Thus the characteristic length $l_{u}$ is given by

$$
l_{u}=\sqrt{\frac{2 E^{\prime} \bar{\delta} h}{\tau_{\mathrm{p}}-\tau_{\mathrm{r}}}}=\sqrt{\frac{2 E^{\prime} h \delta_{95}^{\mathrm{p}}}{3\left(\tau_{\mathrm{p}}-\tau_{\mathrm{r}}\right)}}
$$

so that equivalent values of $\bar{\delta}$ and thus of $l_{u}$ are obtained by taking $\delta \xi_{5}^{\mathrm{p}}$ (exponential degradation) as $1 \cdot 5 \delta_{\mathrm{r}}^{\mathrm{p}}$ (linear degradation).

The algebra for the exponential degradation case is provided in the Appendix, from which the critical condition for catastrophic failure is obtained as

$$
l_{0}=\sqrt{1-r(1-\ln r)} \frac{2 l_{u}}{r_{0}}
$$

This relationship is shown in Fig. 6 for comparison with the corresponding criterion for linear degradation, from which it is clear that, for given values of $r$ and $l_{u}$, the exponential decay gives rise to a more stringent condition on $l_{0}$.

\section{LARGE DEFORMATION FINITE-ELEMENT MODELLING \\ RITSS approach}

The LDFE analyses of catastrophic failure were conducted using an approach termed remeshing and interpolation technique with small strain (RITSS, Hu \& Randolph, 1998; Wang et al., 2010). Here a brief description of the numerical process is provided, and more details may be found in Wang et al. (2010, 2013b, 2015).

The RITSS approach, falling in the category of 'arbitrary Lagrangian-Eulerian' methods, divides the whole analysis into a series of small strain analysis increments to avoid mesh distortion, followed by remeshing and interpolation of all field quantities from old to new meshes. In this study, each small strain increment is invoked by a main program coded in Fortran. In each increment, the Lagrangian calculation is undertaken using the commercial package Abaqus/Standard (Dassault Systems, 2011). Python, the Abaqus built in script, was used to code files controlling the finite-element model and output field variables from the result files to be mapped to the new mesh at the start of the next increment. The series of small steps including remeshing, interpolation and implicit calculations is repeated until the whole analysis is completed. Finite strain theory (i.e. updated Lagrangian calculation) is used during each small step.

The quality of interpolation plays a dominant role in the accuracy of RITSS analysis. The modified unique element method (MUEM) was adopted for this work, the robustness of which has been validated by $\mathrm{Hu} \&$ Randolph (1998) and Wang et al. (2013a, 2015). The field variables, such as stresses, plastic shear strains and material properties are recorded at deformed integration points, which are triangulated via Delaunay triangulation after the Lagrangian calculation of each incremental step. At the commencement of the next step, the field variables at each new integration point are recovered from those at the old triangulation points within which the new integration point is located. For those isolated from any triangular element, we simply recover the field variables from the two nearest old integration points by adopting a weighting value with respect to their distances.

\section{Model details}

Figure 1(b) shows the model used in the numerical study. The model is shaped as a long embankment (representing part of an infinite slope) and comprises three parts: an upper elastic section of the embankment, a central strain-softening elasto-plastic 'weak' layer representing the shear band and a 
lower elastic base. The whole embankment (or slope) is inclined at an angle $\theta$ with both front and rear faces at an angle $\psi$ to the top and bottom faces of the embankment. The softenable layer, located at depth $h$, extends the full length $L$ of the model and covers a thickness $s$. At its centre, a pre-softened 'remoulded' zone of length $l_{0}$, with shear strength, $\tau_{\mathrm{r} 0}=\tau_{\mathrm{r}}$, equal to the residual shear strength outside the pre-softened zone, is introduced at the start of the analysis to represent the initial shear band.

Only the material in the shear band was allowed to deform plastically and undergo strain softening. Driven by the gravity loading applied to the embankment, the initial shear band is expected to propagate along adjacent sections of the weak layer. The gravity force was ramped up in small increments in order to avoid element distortion for each increment, with a condition that if the equivalent plastic strain in any element is observed to be larger than unity, the increment was restarted, halving the previous incremental gravity acceleration. The initial incremental gravity acceleration was set to $1.0 \mathrm{~m} / \mathrm{s}^{2}$.

In order to be implemented into a solid element within Abaqus, the strain-softening governing equations (16) and (33) were expressed in terms of plastic shear strain, with the shear strength expressed as (see Fig. 2(b) and Fig. 7(b))

$$
\begin{aligned}
& \tau=\tau_{\mathrm{p}}+\left(\tau_{\mathrm{r}}-\tau_{\mathrm{p}}\right) \frac{\gamma^{\mathrm{p}}}{\gamma_{\mathrm{r}}^{\mathrm{p}}} \\
& \tau=\tau_{\mathrm{r}}+\left(\tau_{\mathrm{p}}-\tau_{\mathrm{r}}\right) e^{-3 \gamma^{\mathrm{p}} / \gamma_{95}^{\mathrm{p}}}
\end{aligned}
$$

where $\gamma_{\mathrm{r}}^{\mathrm{p}}$ (for linear degradation) is the value of $\gamma^{\mathrm{p}}$ at which the material just reaches the residual state. A modified Tresca model with yield strength adjusted according to equations (37) and (38) was adopted at each sub-increment. The flow rule for the Tresca model together with a high Poisson's ratio ensures essentially constant volume (so undrained) conditions.
Any 'shear lock' effect is expected to be weak for this problem, so full integration rather than reduced integration was adopted to enhance the density of sampling points and hence the quality of interpolation. Equally, the fully integrated, first-order, solid elements in Abaqus/Standard do not suffer from volumetric locking because these elements feature constant volume strain in each element (Dassault Systèmes, 2011). A plane strain element set was used for all numerical cases. Since the material properties are different for each layer, the remeshing and interpolation processes were performed separately in each layer. The geometry and meshes were updated at each increment. However, the strength profile was updated far more frequently (at every sub-increment within each increment) with the maximum sub-increment set to $1 \%$ of each increment.

A typical detail of the mesh in the vicinity of the shear band and the displacement across the shear band at the end of an increment is shown in Fig. 8. Two examples are shown, with the potentially softening layer modelled as a single row, or four rows (each of the same thickness as the single row). As expected, the strain-softening nature of the shear band always concentrates the plastic shearing within a single element row, and analyses using one, two or four 'softenable' rows gave identical results. As such, the main set of analyses was conducted with only a single row of softening elements, of thickness $s$.

Independence of the results from the thickness of the softening layer was achieved by linking the shear strain $\gamma$ across the layer to the displacement $\delta$ in the theoretical analysis through $\delta=\gamma$. As seen in Fig. 9, a set of analyses varying $s$ between $0.0625 \mathrm{~m}$ and $0.5 \mathrm{~m}$, adjusting $G_{\mathrm{s}}$ and $\gamma_{\mathrm{r}}^{\mathrm{p}}$ to maintain consistent values of $l_{u, \mathrm{e}}$ and $\bar{\delta}$, showed very similar results, indicating that a thickness of $0.125 \mathrm{~m}$ was sufficient to avoid any mesh-dependency.

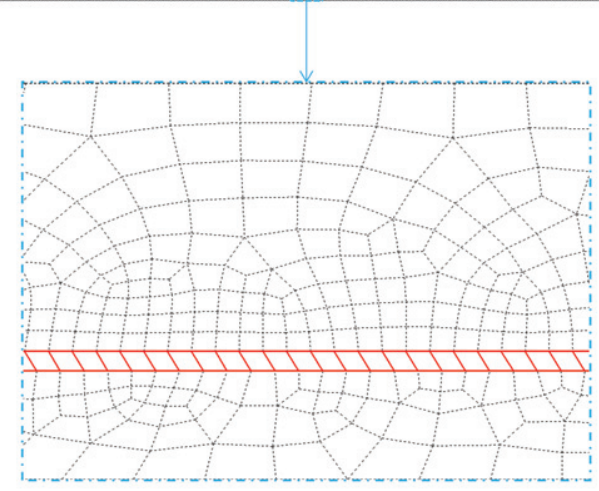

(a)

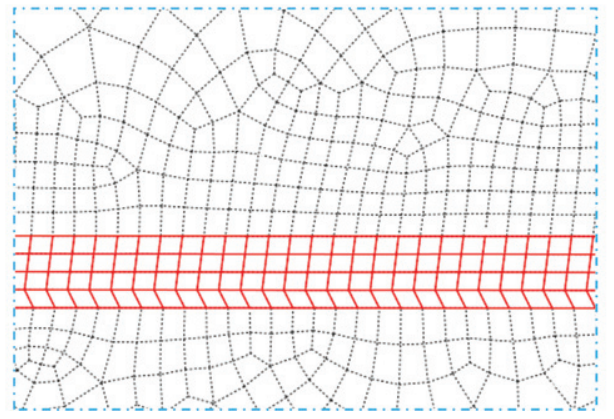

(b)

Fig. 8. Detail of the shear band in numerical study: (a) single row of elements; (b) four rows of elements 


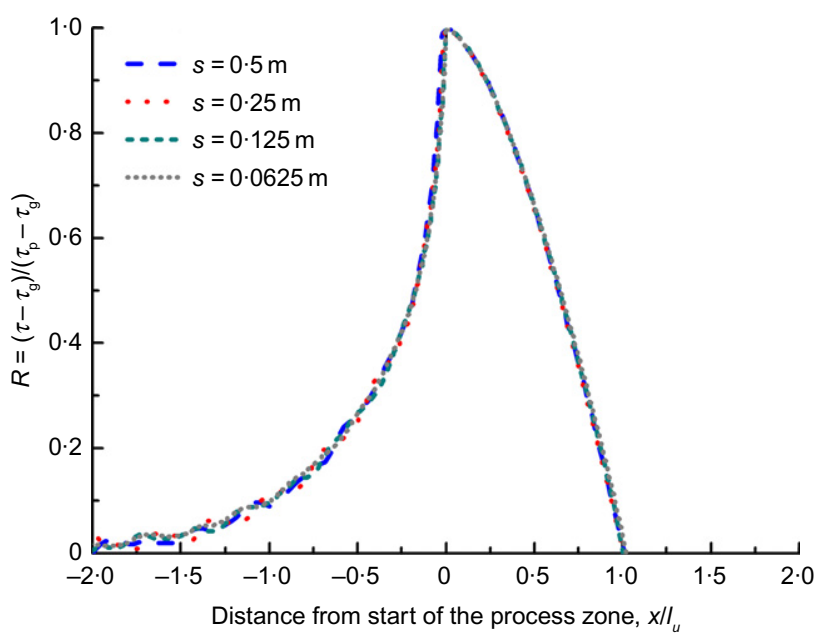

Fig. 9. Influence of mesh size (shear band thickness) on LDFE results with fixed $\bar{\delta}$ and $l_{u, \mathrm{e}}$

\section{Simulation results}

The geometry and base set of material properties adopted for the LDFE analyses are summarised in Table 1 (see Fig. 1(b)). In the analytical analysis the base is assumed to be rigid, but as will be discussed later, the critical values of $r$ are unaffected by whether the base modulus $E_{\mathrm{b}}^{\prime}$ was taken as the same as $E^{\prime}$ or a factor of 1000 times higher. Therefore, plane strain modulus values in the overlying sliding layer $\left(E^{\prime}\right)$ and base layer $\left(E_{\mathrm{b}}^{\prime}\right)$ were initially taken as the same, and consistent with the shear modulus $G_{\mathrm{s}}$ in the softening layer. The value of Poisson ratio was set to 0.495 in order to simulate closely undrained conditions, which results in a modulus ratio, $E^{\prime} / G_{\mathrm{s}}$ close to 4 . A rigidity index $G_{\mathrm{s}} / \tau_{\mathrm{p}}$ of 50 was adopted, together with a base case sensitivity of $S_{\mathrm{t}}=5$. In order to achieve identical values of characteristic displacement $\bar{\delta}(0.025,0.1$ and $0.25 \mathrm{~m})$ for the two different degradation relationships, values of $\gamma_{95}^{\mathrm{p}}$ were taken as $1 \cdot 5 \gamma_{\mathrm{r}}^{\mathrm{p}}$,

Table 1. Base case parameters for LDFE analyses

\begin{tabular}{|c|c|c|}
\hline Parameter & Value & Unit \\
\hline Overall embankment length, $L$ & 700 & $\mathrm{~m}$ \\
\hline Pre-softened zone length, $l_{0}$ & 90 & $\mathrm{~m}$ \\
\hline Overall height, $H$ & 10 & $\mathrm{~m}$ \\
\hline Height of sliding material, $h$ & $7 \cdot 2$ & $\mathrm{~m}$ \\
\hline Shear band thickness, $s$ & $0 \cdot 125$ & \\
\hline Slope angle, $\theta$ & 5 & degrees \\
\hline End-slope angle, $\psi$ & 30 & degrees \\
\hline Poisson's ratio, $v$ & $0 \cdot 495$ & \\
\hline $\begin{array}{l}\text { Young's modulus for plane strain } \\
\text { conditions* } E^{\prime}\end{array}$ & $1 \cdot 98$ & $\mathrm{MPa}$ \\
\hline Shear modulus in softening layer, $G_{\mathrm{s}}$ & 500 & $\mathrm{kPa}$ \\
\hline $\begin{array}{l}\text { Plane strain Young's modulus for } \\
\text { base layer, } E_{\mathrm{b}}^{\prime}\end{array}$ & $E^{\prime}$ or $1000 E^{\prime}$ & \\
\hline Peak shear strength, $\tau_{\mathrm{p}}$ & 10 & $\mathrm{kPa}$ \\
\hline Residual shear strength, $\tau_{\mathrm{r}}$ & $\begin{array}{l}1 \cdot 25,2 \\
\quad \text { and } 3 \cdot 33\end{array}$ & $\mathrm{kPa}$ \\
\hline $\begin{array}{l}\text { Plastic shear strain to residual, } \gamma_{\mathrm{r}}^{\mathrm{p}} \\
\text { (linear decay) }\end{array}$ & $\begin{array}{l}0 \cdot 4,1 \cdot 6 \\
\quad \text { and } 4\end{array}$ & \\
\hline $\begin{array}{l}\text { Plastic shear strain to } 95 \% \text { reduction } \\
\text { in strength, } \gamma_{95}^{p} \text { (exponential decay) }\end{array}$ & $0 \cdot 6,2 \cdot 4$ and & \\
\hline $\begin{array}{l}\text { Characteristic displacement in the } \\
\text { process zone, } \bar{\delta}\end{array}$ & $\begin{array}{l}0 \cdot 025,0 \cdot 1 \\
\quad \text { and } 0 \cdot 25\end{array}$ & \\
\hline Submerged density of soil, $\rho$ & 600 & $\mathrm{~kg} / \mathrm{m}^{3}$ \\
\hline
\end{tabular}

*For plane strain conditions, $E^{\prime}=E /\left(1-v^{2}\right)$, where $E$ is the uniaxial Young's modulus.

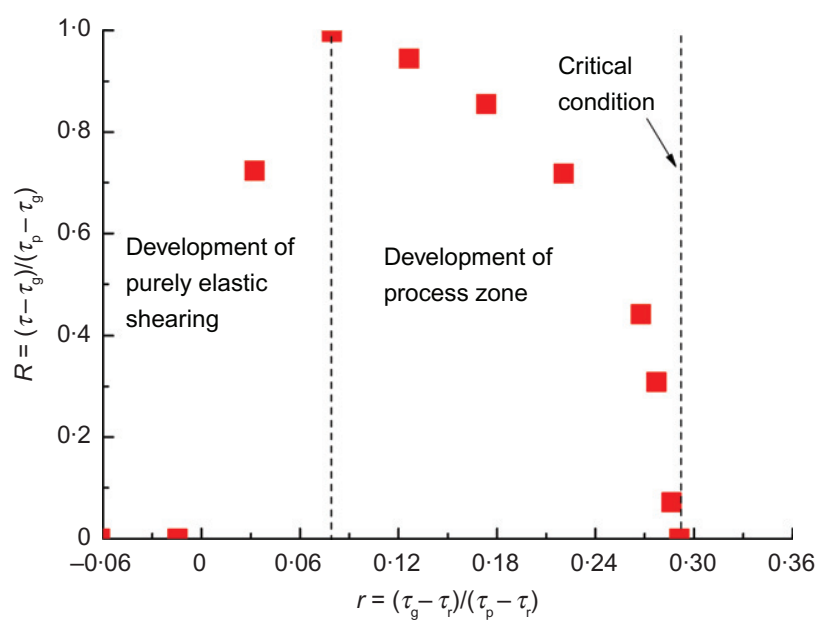

Fig. 10. Normalised shear stress at point $x_{1}$ under different levels of gravity load for typical case, $\bar{\delta}=0 \cdot 1 \mathrm{~m}\left(\gamma_{\mathrm{r}}^{\mathrm{p}}=1 \cdot 6\right)$

with analyses undertaken for $\gamma_{\mathrm{r}}^{\mathrm{p}}$ equal to $0 \cdot 4,1.6$ and 4 , and correspondingly $\gamma_{95}^{\mathrm{p}}$ values of $0 \cdot 6,2 \cdot 4$ and 6 . For these values, the characteristic lengths for both linear and exponential degradations are $l_{u, \mathrm{e}}=1.9 \mathrm{~m}$, and $l_{u}=9.4,18.9$ and $29.9 \mathrm{~m}$, respectively. Below, results for the case of linear degradation with $\bar{\delta}=0 \cdot 1 \mathrm{~m}\left(\gamma_{\mathrm{r}}^{\mathrm{p}}=1 \cdot 6\right)$ are presented in detail to illustrate the evolution of catastrophic failure.

As shown in Fig. 4, approach of the critical condition is indicated by the shear stress level at the point $x=x_{1}$ (i.e. at the start of the pre-softened zone). To distinguish different stages, the shear stresses at point $\boldsymbol{x}_{1}$ under different levels of gravity load are plotted in Fig. 10 for the selected case with $\delta=0.1 \mathrm{~m}\left(\gamma_{\mathrm{r}}^{\mathrm{p}}=1 \cdot 6\right)$. The gravity load is calculated as

$$
\tau_{\mathrm{g}}=\rho \boldsymbol{g}_{\mathrm{acc}} h \sin \theta
$$

where $\boldsymbol{g}_{\text {acc }}$ is the accumulated gravity acceleration at the current step, and normalised as $r$ (see equation (2)), while the shear stress at point $x_{1}$ is normalised as $R$ (see equation (27)). The value of $R$ starts from 0 and grows to 1 at the point where the peak shear stress is mobilised adjacent to the pre-softened zone. The ratio then gradually drops to 0 , which marks the critical condition.

It is found from Fig. 10 that the process zone is initiated at around $r=0.079\left(g_{\text {acc }}=7.0 \mathrm{~m} / \mathrm{s}^{2}\right)$ and catastrophic failure occurs at $r=0.293\left(g_{\text {acc }}=11.53 \mathrm{~m} / \mathrm{s}^{2}\right)$. Therefore, the three stages of progressive failure are identified: the first is purely elastic shearing, $r \leq 0.079$; the second is development of the process zone, $0.079<r<0.293$; the third is catastrophic failure, $r \geq 0 \cdot 293$. In the following, four characteristic points are chosen, $r=-0 \cdot 015,0 \cdot 079,0.221$ and 0.293 (correspondingly, $\boldsymbol{g}_{\text {acc }}=5 \cdot 0,7 \cdot 0,10 \cdot 0$ and $11 \cdot 53 \mathrm{~m} / \mathrm{s}^{2}$ ), to investigate incremental displacement fields and shear stress distributions within the shear band, to explore the evolution of failure.

Figure 11 and Fig. 12, respectively, show the normalised incremental displacement fields (for each gravity increment of $0.1 \mathrm{~m} / \mathrm{s}^{2}$ ) and normalised shear stress distributions within the shear band at various loading stages (i.e. $r=-0 \cdot 015$, $0.079,0.221$ and 0.293 ). The incremental displacement is normalised by the shear band thickness, $s$. Since the actual ratio of height to width is rather small $(0 \cdot 02)$, it is enlarged five times for Fig. 11. During purely elastic shearing $\left(r \leq 0.079\right.$ or $\left.g_{\text {acc }} \leq 7.0 \mathrm{~m} / \mathrm{s}^{2}\right)$, the incremental displacement is small and concentrated in the material overlying the pre-softened zone. The shear stress increases from $\tau_{\mathrm{g}}$ to peak (correspondingly $R$ increases from 0 to 1 ) in front of the pre-softened zone (Fig. 12), showing no softening occurs at that stage. 
After the initiation of the process zone $\left(r>0.079\right.$ or $g_{\text {acc }}>$ $\left.7 \cdot 0 \mathrm{~m} / \mathrm{s}^{2}\right)$, the rate of displacement increases and propagates into process zones at each end of the pre-softened zone. Meanwhile, the soil in the process zones starts to strain soften

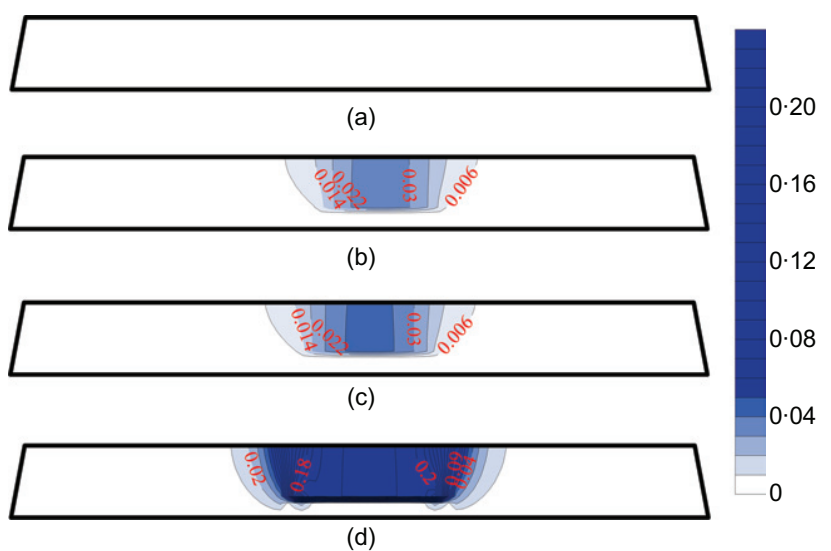

Fig. 11. Incremental displacements at different loading stages normalised by $s, \bar{\delta}=0.1 \mathrm{~m}\left(\gamma_{\mathrm{r}}^{\mathrm{p}}=1 \cdot 6\right)$ : (a) $r=-0.015$; (b) $r=0.079$; (c) $r=0.221$; (d) $r=0.293$

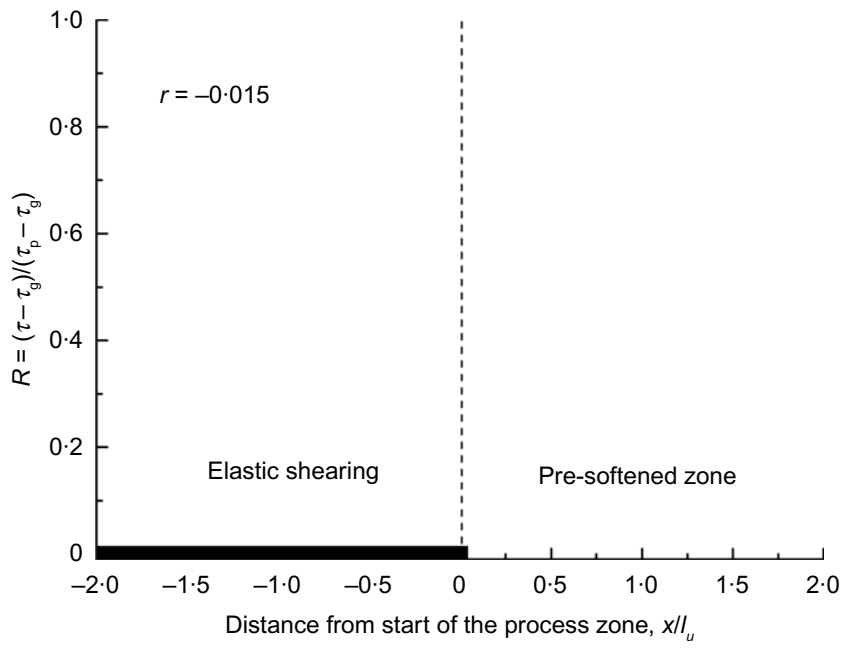

(a)

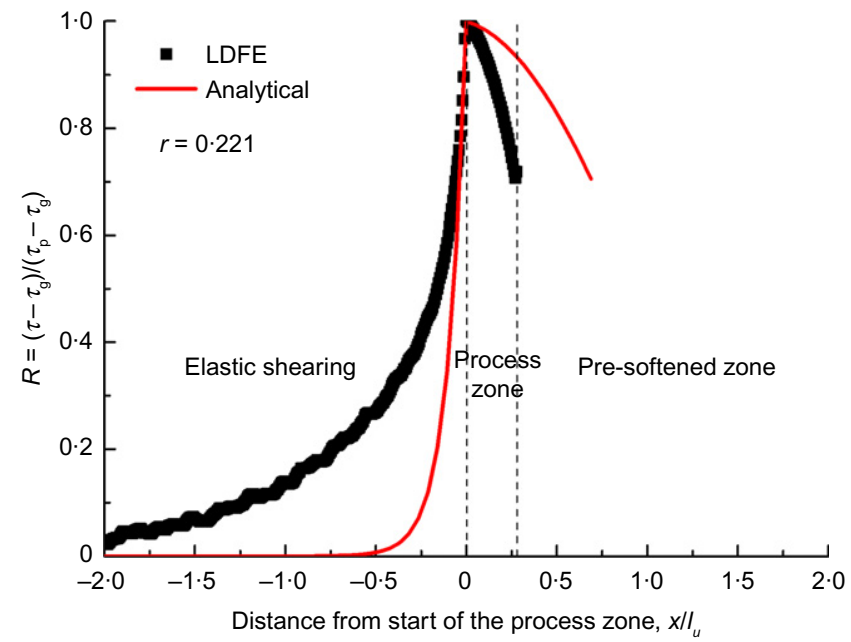

(c) with the shear stress reducing from the peak strength; in turn this results in further increase in displacement rate of the overlying soil, and outward propagation of the elastic zone. This cycle is repeated until catastrophic failure is finally triggered at $r=0.293\left(g_{\text {acc }}=11.53 \mathrm{~m} / \mathrm{s}^{2}\right)$.

It is evident from Fig. 12 that the shapes of the profiles from the LDFE analyses do not match the analytical solution, with the width of the process zone overestimated, and the decay of elastic shear stress too abrupt. The discrepancy arises from the simplified assumption of equation (8) compared with equation (32), as discussed previously. This results in the analytical solutions overestimating the size of the process zone (as shown later in Table 3). The more gradual decay of shear stress for elastic deformation of the shear band also explains why the normalised gravity load $\left(r_{\mathrm{e}}\right)$ at the initiation of the process zone predicted analytically is lower than the LDFE value.

In order to explore the discrepancy between the analytical and LDFE shear stress profiles, a series of analyses were undertaken that progressively approach the assumptions in the analytical solution, which ignores any deformation in the base layer, or shear deformation $\partial \delta / \partial y$ (where $y$ is the quasi-vertical coordinate normal to the layer) in the sliding layer. First the base stiffness was increased by a factor of 1000 relative to $E^{\prime}$; then the ratio $E^{\prime} / G_{\mathrm{s}}$ was increased

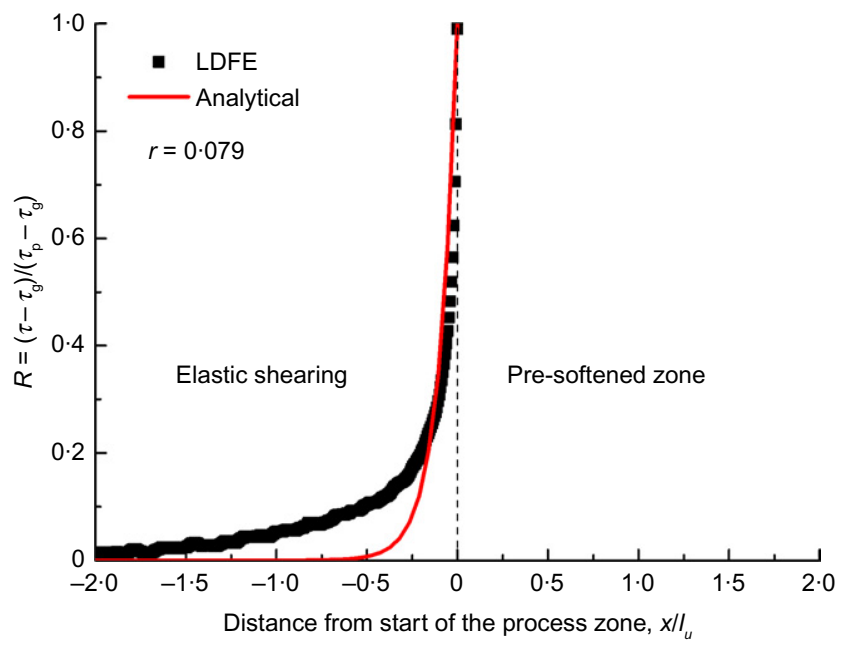

(b)

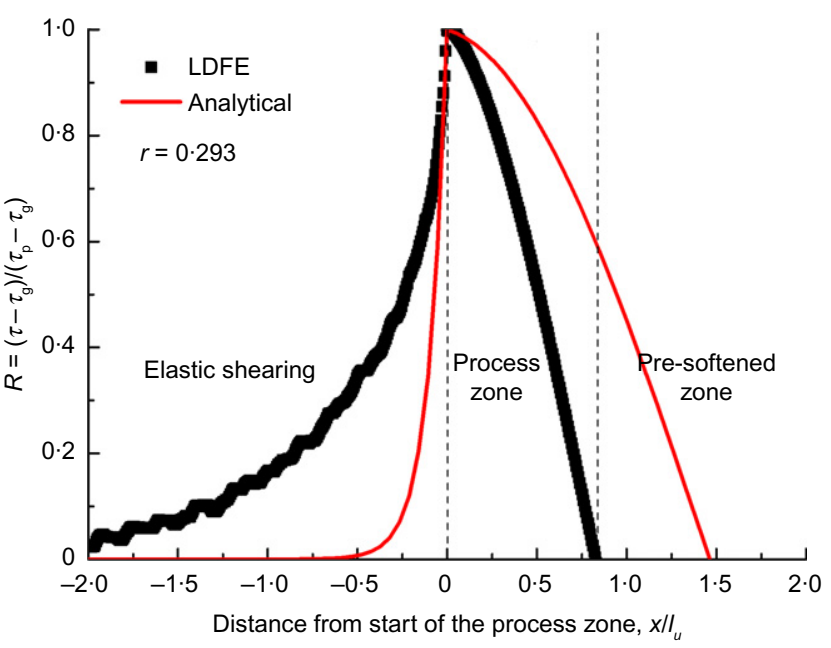

(d)

Fig. 12. Distributions of the normalised shear stress at different loading stages for the typical case, $\bar{\delta}=0 \cdot 1 \mathrm{~m}\left(\gamma_{\mathrm{r}}^{\mathrm{p}}=1 \cdot 6\right):(\mathrm{a}) r=-0 \cdot 015$; (b) $r=0 \cdot 079$; (c) $r=0 \cdot 221$; (d) $r=0 \cdot 293$ 
Table 2. Parametric study with respect to the influence of $E^{\prime} / G_{\mathrm{s}}$ (linear degradation)

\begin{tabular}{|c|c|c|c|c|c|c|c|c|c|}
\hline \multirow[t]{2}{*}{$E^{\prime} / G_{\mathrm{s}}$} & \multirow[t]{2}{*}{$E_{\mathrm{b}}^{\prime} / E^{\prime}$} & \multirow[t]{2}{*}{$G_{\mathrm{s}} / \tau_{\mathrm{p}}$} & \multirow[t]{2}{*}{$l_{u, \mathrm{e}} / l_{0}$} & \multirow[t]{2}{*}{$l_{u} / l_{0}$} & \multirow[t]{2}{*}{$\beta^{*}$} & \multicolumn{2}{|c|}{$\omega / l_{u}$} & \multicolumn{2}{|c|}{ Critical value of $r \dot{ }$} \\
\hline & & & & & & LDFE & Analytical & LDFE & Analytical \\
\hline 3.96 & 1 & 10 & $0 \cdot 021$ & $0 \cdot 094$ & 0.975 & 0.628 & $1 \cdot 311$ & $0 \cdot 155$ & $0 \cdot 158$ \\
\hline 3.96 & 1000 & 10 & $0 \cdot 021$ & $0 \cdot 094$ & 0.975 & $0 \cdot 628$ & $1 \cdot 311$ & $0 \cdot 155$ & $0 \cdot 158$ \\
\hline $39 \cdot 6$ & 1000 & 10 & $0 \cdot 066$ & $0 \cdot 297$ & 0.975 & $1 \cdot 080$ & $1 \cdot 311$ & $0 \cdot 371$ & 0.372 \\
\hline 118.8 & 1000 & 10 & $0 \cdot 115$ & $0 \cdot 514$ & 0.975 & $1 \cdot 241$ & $1 \cdot 311$ & $0 \cdot 505$ & 0.507 \\
\hline $198 \cdot 0$ & 1000 & 10 & $0 \cdot 148$ & 0.663 & 0.975 & $1 \cdot 275$ & $1 \cdot 311$ & $0 \cdot 569$ & $0 \cdot 57$ \\
\hline
\end{tabular}

$* \beta=\sqrt{1-\frac{\left(\tau_{\mathrm{p}}-\tau_{\mathrm{r}}\right) s}{G_{\mathrm{s}} \delta_{\mathrm{r}}^{\mathrm{p}}}}$

$\dagger r=\frac{\tau_{\mathrm{g}}-\tau_{\mathrm{r}}}{\tau_{\mathrm{p}}-\tau_{\mathrm{r}}}$

Table 3. Parameters and results (linear degradation)

\begin{tabular}{|c|c|c|c|c|c|c|c|c|}
\hline \multirow[t]{2}{*}{$\bar{\delta} / l_{0}: \%$} & \multirow[t]{2}{*}{$S_{\mathrm{t}}$} & \multirow[t]{2}{*}{$l_{u, \mathrm{e}} / l_{0}$} & \multirow[t]{2}{*}{$l_{u} / l_{0}$} & \multirow[t]{2}{*}{$\beta^{*}$} & \multicolumn{2}{|c|}{$\omega / l_{u}$} & \multicolumn{2}{|c|}{ Critical value of $r \dagger$} \\
\hline & & & & & LDFE & Analytical & LDFE & Analytical \\
\hline $0 \cdot 028$ & 3 & $0 \cdot 021$ & $0 \cdot 115$ & $0 \cdot 983$ & $0 \cdot 464$ & $1 \cdot 364$ & $0 \cdot 183$ & $0 \cdot 187$ \\
\hline $0 \cdot 11$ & 3 & $0 \cdot 021$ & $0 \cdot 230$ & 0.996 & $0 \cdot 872$ & 1.473 & $0 \cdot 313$ & $0 \cdot 315$ \\
\hline $0 \cdot 278$ & 3 & $0 \cdot 021$ & $0 \cdot 363$ & 0.998 & 0.948 & $1 \cdot 511$ & $0 \cdot 421$ & $0 \cdot 421$ \\
\hline 0.028 & 5 & $0 \cdot 021$ & $0 \cdot 105$ & 0.980 & 0.5 & $1 \cdot 342$ & $0 \cdot 171$ & $0 \cdot 173$ \\
\hline $0 \cdot 11$ & 5 & $0 \cdot 021$ & $0 \cdot 210$ & 0.995 & 0.833 & $1 \cdot 463$ & 0.293 & $0 \cdot 296$ \\
\hline $0 \cdot 278$ & 5 & $0 \cdot 021$ & 0.332 & 0.998 & $1 \cdot 10$ & $1 \cdot 504$ & 0.399 & 0.399 \\
\hline 0.028 & 8 & 0.021 & $0 \cdot 100$ & 0.979 & $0 \cdot 399$ & $1 \cdot 330$ & $0 \cdot 165$ & $0 \cdot 167$ \\
\hline $0 \cdot 11$ & 8 & 0.021 & $0 \cdot 201$ & 0.995 & 0.796 & $1 \cdot 458$ & $0 \cdot 283$ & $0 \cdot 286$ \\
\hline $0 \cdot 278$ & 8 & $0 \cdot 021$ & $0 \cdot 317$ & 0.998 & 0.981 & $1 \cdot 511$ & $0 \cdot 386$ & $0 \cdot 388$ \\
\hline
\end{tabular}

$* \beta=\sqrt{1-\frac{\left(\tau_{\mathrm{p}}-\tau_{\mathrm{r}}\right) s}{G_{\mathrm{s}} \delta_{\mathrm{r}}^{\mathrm{p}}}}$

$\dagger r=\frac{\tau_{\mathrm{g}}-\tau_{\mathrm{r}}}{\tau_{\mathrm{p}}-\tau_{\mathrm{r}}}$

gradually from 1 to 50 . All these analyses gave identical critical gravity loading between LDFE and analytical solutions, as shown in Table 2. However, as shown in Fig. 13, the shear stress profiles gradually converged to the analytical solution. In fact, the base stiffness (either identical modulus to the sliding layer, or essentially rigid) has a rather small effect, whereas the relative shear stiffness of the sliding and softening layers has a major effect on the detailed shear stress profiles, but no effect on the critical condition for catastrophic failure. Also as shown in Table 2, both the rigid base $\left(E_{\mathrm{b}}^{\prime} / E^{\prime}=1000\right)$ and homogeneous base cases lead to the identical value of $r$, illustrating that the assumption of a stiff base in current analytical analysis and previous works (Palmer \& Rice 1973; Puzrin et al., 2004; Puzrin \& Germanovich, 2005) is not physically necessary.

Comparison with analytical solutions - linear degradation. A parametric study was conducted, varying the soil sensitivity $S_{\mathrm{t}}$ from 3 to 8 and the characteristic displacement $\bar{\delta}$ from 0.025 to $0.25 \mathrm{~m}$. Table 3 lists the parameters and simulation results for all cases of linear degradation, while Fig. 14(a) presents the critical values of the normalised gravity load $r$ for both LDFE results and analytical solutions, that is, equation (30) taking $r_{0}=r$, with respect to the influence of $\delta$. The LDFE and analytical values of $r$ for catastrophic failure agree well with each other, and both increase with the value of $\bar{\delta}$. The analytical values of the critical $r$ are marginally higher than the LDFE values, but the differences (from 0 to $2 \%$ ) are within any likely error

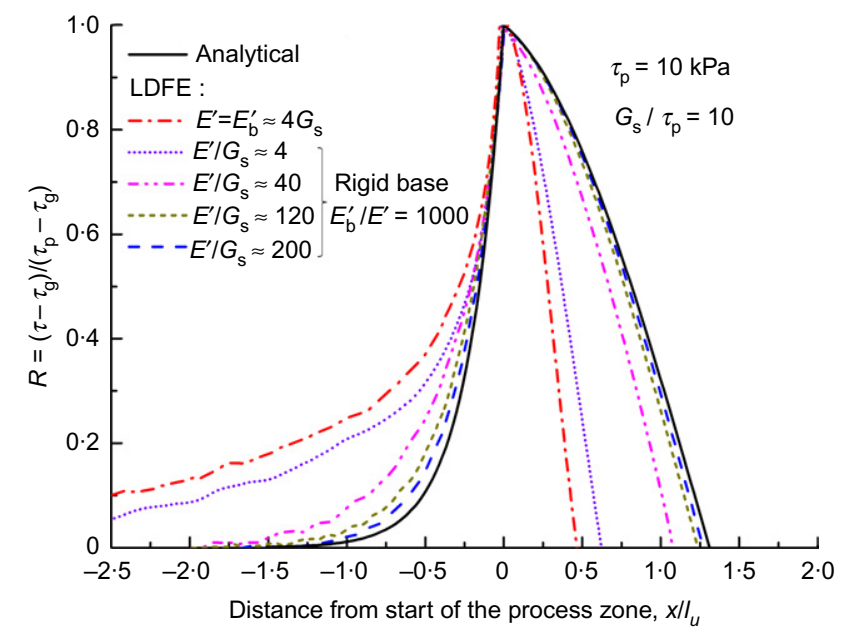

Fig. 13. Distributions of normalised shear stress within shear band at the critical condition with respect to the influence of $E^{\prime} / G_{\mathrm{s}}$ (linear degradation)

bound for the numerical analyses. As noted previously, the critical values of $r$ were found to be unaffected by whether the base modulus $E_{\mathrm{b}}^{\prime}$ was taken the same as $E^{\prime}$ or a factor of 1000 times higher.

The value of $r$ at initiation of the process zone is independent of $\bar{\delta}$ (since only elastic shearing occurs), with equation (15), taking $r_{0, \mathrm{e}}=r_{\mathrm{e}}$, predicting a value of $r_{\mathrm{e}}=0.04$. The value from the LDFE analyses is 0.079 , which is 
consistent with the more gradual decay of shear stress in the elastic zone, as shown in Fig. 12.

Comparison with analytical solutions - exponential degradation.

Table 4 shows the parameters and simulation results for all cases of exponential degradation, while Fig. 14(b) shows the

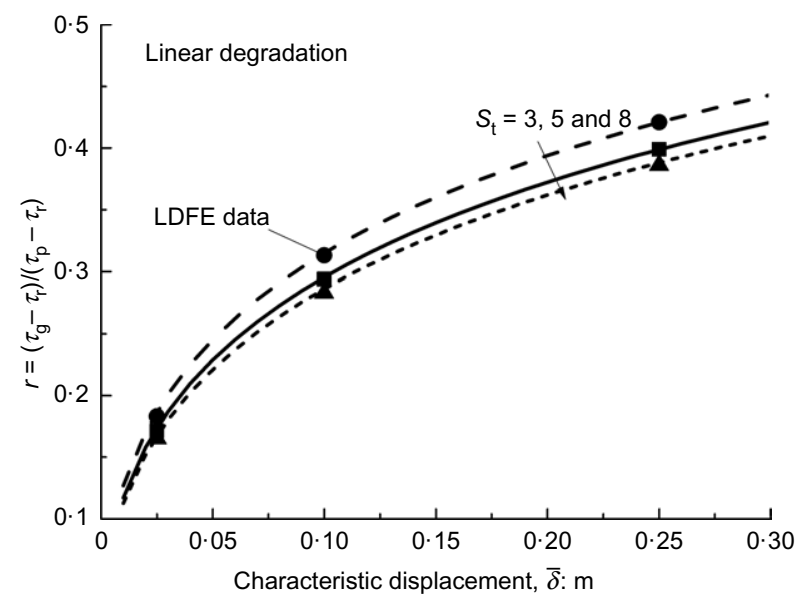

(a)

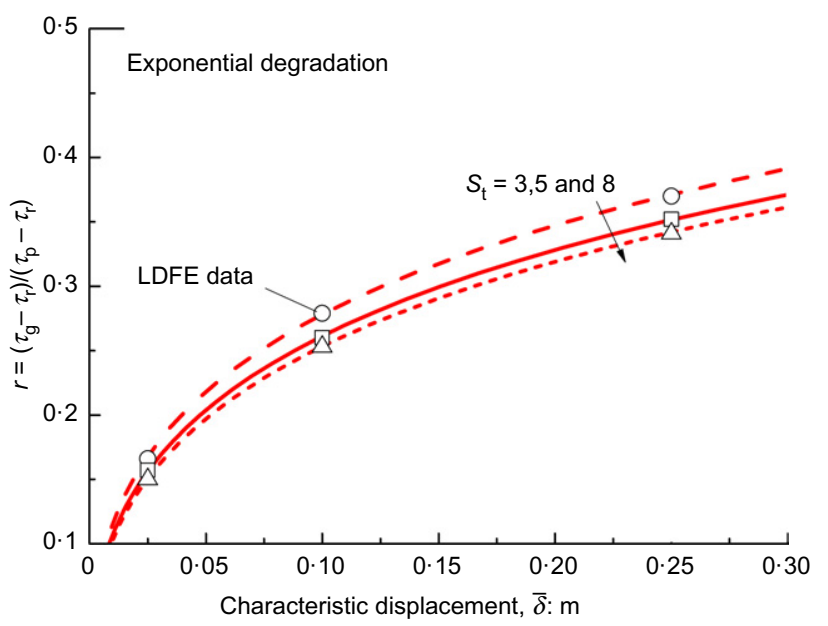

(b)

Fig. 14. Comparison of the normalised gravity load $\boldsymbol{r}$ for the critical condition: (a) linear degradation; (b) exponential degradation critical values of normalised gravity load $r$ for both LDFE results and analytical solutions, that is, equation (36) taking $r_{0}=r$, with respect to the influence of $\bar{\delta}$.

As for the case of linear degradation, the analytical values of critical $r$ agree well with but are (mostly) marginally higher than the LDFE values. The process zone length $\omega$ is overestimated and correspondingly the elastic contribution is underestimated by the analytical solutions compared with LDFE analyses, which is consistent with the observations for linear degradation.

For a given value of $\bar{\delta}$ (and hence $l_{u}$ ), the critical values of $r$ under exponential degradation are lower than those under linear degradation. For example, with $\bar{\delta}=0 \cdot 1 \mathrm{~m}$ and $S_{\mathrm{t}}=5$, the LDFE critical values of $r$ for exponential degradation and linear degradation are 0.260 and 0.293 , respectively. Equally, for any given value of $r$, the exponential degradation leads to a lower value of critical length $l_{0}$ of the pre-softened zone (as shown in Fig. 6).

The apparent conservatism of the exponential strainsoftening compared with linear degradation suggests that the linear degradation criterion of equation (30) does not capture the full effects of different degradation laws. Critical conditions are influenced by the initial rate of post-peak degradation, in addition to the characteristic displacement $\bar{\delta}$. For a given value of $\bar{\delta}$, the initial post-peak rate of degradation for the exponential law is twice that for linear degradation. Even so, the difference in calculated critical values of $r$ or $l_{0}$ is still only 10 to $15 \%$.

\section{CONCLUSIONS}

This study has explored the critical conditions and developed propagation criteria for catastrophic failure of an infinite slope in undrained sensitive clay as a result of the formation of an initial fully softened shear band in a thin lens of weaker material. In some practical cases this shear band initiation scenario can be more relevant than the conventional one of progressive growth of the initial shear band in a homogeneous material. The framework of the 'process zone approach', which was proposed by Puzrin \& Germanovich (2005) and took the significance of process zones at each end of an initial 'pre-softened' region into account, has been extended to consider the role of elastic deformations within the shear band and in the entire sliding layer. Two different strain-softening relationships, giving linear and exponential strength degradation with increasing plastic shear strain, have been considered, and new analytical solutions derived

Table 4. Parameters and results (exponential degradation)

\begin{tabular}{|c|c|c|c|c|c|c|c|c|}
\hline \multirow[t]{2}{*}{$\bar{\delta} / l_{0}: \%$} & \multirow[t]{2}{*}{$S_{\mathrm{t}}$} & \multirow[t]{2}{*}{$l_{u, \mathrm{e}} / l_{0}$} & \multirow[t]{2}{*}{$l_{u} / l_{0}$} & \multirow[t]{2}{*}{$\kappa^{*}$} & \multicolumn{2}{|c|}{$\omega / l_{u}$} & \multicolumn{2}{|c|}{ Critical value of $r \dagger$} \\
\hline & & & & & LDFE & Analytical & LDFE & Analytical \\
\hline $0 \cdot 028$ & 3 & $0 \cdot 021$ & $0 \cdot 115$ & $0 \cdot 067$ & 0.687 & $1 \cdot 453$ & $0 \cdot 166$ & $0 \cdot 168$ \\
\hline $0 \cdot 11$ & 3 & $0 \cdot 021$ & $0 \cdot 230$ & $0 \cdot 017$ & 0.919 & $1 \cdot 468$ & $0 \cdot 279$ & $0 \cdot 278$ \\
\hline $0 \cdot 278$ & 3 & $0 \cdot 021$ & $0 \cdot 363$ & 0.007 & $0 \cdot 865$ & 1.423 & $0 \cdot 370$ & $0 \cdot 371$ \\
\hline $0 \cdot 028$ & 5 & $0 \cdot 021$ & $0 \cdot 105$ & $0 \cdot 08$ & $0 \cdot 636$ & $1 \cdot 448$ & $0 \cdot 157$ & $0 \cdot 156$ \\
\hline $0 \cdot 11$ & 5 & $0 \cdot 021$ & $0 \cdot 210$ & $0 \cdot 02$ & $0 \cdot 837$ & $1 \cdot 456$ & $0 \cdot 260$ & $0 \cdot 261$ \\
\hline $0 \cdot 278$ & 5 & $0 \cdot 021$ & $0 \cdot 332$ & $0 \cdot 008$ & $0 \cdot 871$ & $1 \cdot 429$ & $0 \cdot 352$ & $0 \cdot 352$ \\
\hline $0 \cdot 028$ & 8 & $0 \cdot 021$ & $0 \cdot 100$ & $0 \cdot 088$ & $0 \cdot 643$ & $1 \cdot 420$ & $0 \cdot 150$ & $0 \cdot 151$ \\
\hline $0 \cdot 11$ & 8 & $0 \cdot 021$ & $0 \cdot 201$ & $0 \cdot 022$ & $0 \cdot 842$ & $1 \cdot 461$ & $0 \cdot 253$ & $0 \cdot 253$ \\
\hline $0 \cdot 278$ & 8 & $0 \cdot 021$ & $0 \cdot 317$ & 0.009 & $0 \cdot 911$ & $1 \cdot 427$ & $0 \cdot 341$ & $0 \cdot 342$ \\
\hline
\end{tabular}

$*_{\kappa}=\frac{3\left(\Delta \tau_{\mathrm{p}}-\Delta \tau_{\mathrm{r}}\right) s}{G_{\mathrm{s}} \delta_{95}^{\mathrm{p}}}$

$\dagger r=\frac{\tau_{\mathrm{g}}-\tau_{\mathrm{r}}}{\tau_{\mathrm{p}}-\tau_{\mathrm{r}}}$ 
for the critical length of the pre-softened zone that would result in catastrophic failure. The analytical solutions have been compared with corresponding results from LDFE analysis.

As the normalised gravity load $r$ is increased, three stages may be identified, quantified by values of the characteristic length $l_{u}$, and an equivalent 'elastic' characteristic length $l_{u, \mathrm{e}}$ (equations (3) and (13))

(a) purely elastic deformations within the shear band (shear stress less than the peak shear strength) at each end of the pre-softened zone, with $l_{0} \leq(1-r)\left(2 l_{u, \mathrm{e}} / r_{0}\right)$

(b) development of (plastic) process zones, with $(1-r)$ $\left(2 l_{u, \mathrm{e}} / r_{0}\right)<l_{0}<(1-r)\left(2 l_{u} / r_{0}\right)$

(c) catastrophic failure, with $l_{0} \geq(1-r)\left(2 l_{u} / r_{0}\right)$.

In spite of the fact that for low stiffness ratios, $E^{\prime} / G_{\mathrm{s}}$, analytical estimations of the length of the process zones (at the critical condition), and also the shear stress profile in the region of purely elastic shearing, gave poor agreement with the LDFE results, the analytical and LDFE results always show extremely good agreement with respect to the critical (transition between stage 2 and stage 3 ) value of normalised gravity loading $r$ under given length $l_{0}$ of the pre-softened zone, for both degradation relationships. Apparently, the errors involved in over-simplification of the elastic compression within the material overlying the shear band compensate each other, resulting in a robust solution.

The most significant conclusions from this study are listed below.

(a) The analytical treatment of conditions arising from the proposed shear band initiation scenario, where the initial shear band is formed within the fully softened zone of a weaker material, provides explicit expressions for the critical length of the weak zone for the catastrophic shear band propagation.

(b) The criteria for catastrophic shear band propagation show that some existing criteria, which consider more conventional shear band initiation and progressive growth within homogeneous material, are nonconservative, even for the case of the same residual shear strength inside and outside the weak zone.

(c) Between the linear and exponential shear strength degradation, the latter is considered more representative of actual soil behaviour and also leads to a more conservative evaluation of conditions for catastrophic failure, resulting in magnitudes of critical fully softened length of the band that are 10 to $15 \%$ lower than equivalent values for linear degradation.

(d) In general, the focus on criteria based on the length of fully softened shear band, rather than the overall length including the two process zones, provides a more robust approach, less sensitive both to the simplifying assumptions of process zone based approaches and to inevitable errors in estimating the true length of the process zones.

(e) Numerical validation of the new criteria and underlying assumptions justifies their application within the simplified shear band propagation approach to slope stability problems where the weak zone initiation is a dominating scenario.

\section{ACKNOWLEDGEMENTS}

The research presented here was supported by the Australian Research Council through an ARC Discovery grant (DP120102987). The work forms part of the activities of the Centre for Offshore Foundation Systems (COFS), currently supported as a node of the Australian Research Council Centre of Excellence for Geotechnical Science and Engineering and through the Fugro Chair in Geotechnics, the Lloyd's Register Foundation Chair and Centre of Excellence in Offshore Foundations and the Shell EMI Chair in Offshore Engineering. Alexander Puzrin is grateful to the Institute of Advanced Studies at the University of Western Australia for awarding him a Gledden Fellowship, which helped to initiate this work.

\section{APPENDIX. ALGEBRA FOR EXPONENTIAL DEGRADATION}

For the exponential degradation of shear strength with displacement across the shear band, as given by equation (33) and Fig. 7, the force and displacement relationships in the overlying soil layer may be written

$$
\begin{aligned}
& \frac{\mathrm{d} \Delta \hat{P}}{\mathrm{~d} \hat{x}}=-r+\mathrm{e}^{-2 \hat{\delta}^{\mathrm{p}}} \\
& \frac{\mathrm{d} \hat{\delta}^{\mathrm{p}}}{\mathrm{d} \hat{x}}\left[1-\kappa \mathrm{e}^{-2 \hat{\delta}^{\mathrm{p}}}\right]=\Delta \hat{P} \\
& \kappa=\frac{3\left(\Delta \tau_{\mathrm{p}}-\Delta \tau_{\mathrm{r}}\right) s}{G_{\mathrm{s}} \delta_{95}^{\mathrm{p}}}=2\left(\frac{l_{u, \mathrm{e}}}{l_{u}}\right)^{2}
\end{aligned}
$$

where $\Delta \hat{P}=\Delta P / l_{u}\left(\tau_{\mathrm{p}}-\tau_{\mathrm{r}}\right), \hat{x}=x / l_{u}$ and $\hat{\delta}^{\mathrm{p}}=\delta^{\mathrm{p}} / 2 \bar{\delta}$. Combining equations (40) and (41) yields

$$
\frac{\mathrm{d} \Delta \hat{P}}{\mathrm{~d} \hat{x}}=\frac{\mathrm{d} \Delta \hat{P}}{\mathrm{~d} \hat{\hat{\delta}^{\mathrm{p}}}} \frac{\mathrm{d} \hat{\delta}^{\mathrm{p}}}{\mathrm{d} \hat{x}}=\frac{\mathrm{d} \Delta \hat{P}}{\mathrm{~d} \hat{\delta}^{\mathrm{p}}} \frac{\Delta \hat{P}}{1-\kappa \mathrm{e}^{-2 \hat{\delta}^{p}}}=-r+\mathrm{e}^{-2 \hat{\delta}^{\mathrm{p}}}
$$

At point $\hat{x}=0$

$$
\left.\hat{\delta}^{\mathrm{p}}\right|_{\hat{x}=0}=0,\left.\quad \Delta \hat{P}\right|_{\hat{x}=0}=\frac{\left.\Delta P\right|_{x=0}}{l_{u}\left(\tau_{\mathrm{p}}-\tau_{\mathrm{r}}\right)}=(1-r) \sqrt{\frac{\kappa}{2}}
$$

where $\left.\Delta P\right|_{x=0}=\Delta \tau_{\mathrm{p}} \mathrm{l}_{u}^{\mathrm{e}}$ can be obtained from the elastic zone (see equation (11) with $x=0$ ). Solving equation (43) with the above boundary conditions yields

$$
\Delta \hat{P}=\sqrt{\frac{\kappa}{2}\left(r-\mathrm{e}^{-2 \widehat{\delta}^{\mathrm{p}}}\right)^{2}+1-2 r \hat{\delta}^{\mathrm{p}}-\mathrm{e}^{-2 \hat{\delta}^{\mathrm{p}}}}
$$

According to the instability condition, $\left.\mathrm{d} \Delta \hat{P}\right|_{\hat{x}=\hat{x}_{1}}=0$ and thus

$$
\left.\hat{\delta}^{\mathrm{p}}\right|_{\hat{x}=\hat{x}_{1}}=-\frac{\ln r}{2}
$$

Meanwhile

$$
\left.\Delta \hat{P}\right|_{x=\hat{x}_{1}}=\frac{-\Delta \tau_{\mathrm{r}} l_{0}}{2 l_{u}\left(\tau_{\mathrm{p}}-\tau_{\mathrm{r}}\right)}=\frac{r_{0} \hat{l}_{0}}{2}
$$

where $\hat{l}_{0}=l_{0} / l_{u}$. Substitution of equations (46) and (47) into equation (45) yields

$$
\frac{r_{0}}{2} \hat{l}_{0}=\sqrt{1-r(1-\ln r)}
$$

Therefore, the propagation criterion based on $l_{0}$ under exponential decay is given by

$$
l_{0}=\sqrt{1-r(1-\ln r)} \frac{2 l_{u}}{r_{0}}
$$

The full length of the shear band is given by

$$
\hat{l}=2 \hat{\omega}+\hat{l}_{0}
$$

where $\hat{l}=l / l_{u}$ and $\hat{\omega}=\omega / l_{u}$. Thus, the propagation criterion based on $l$ is given by 


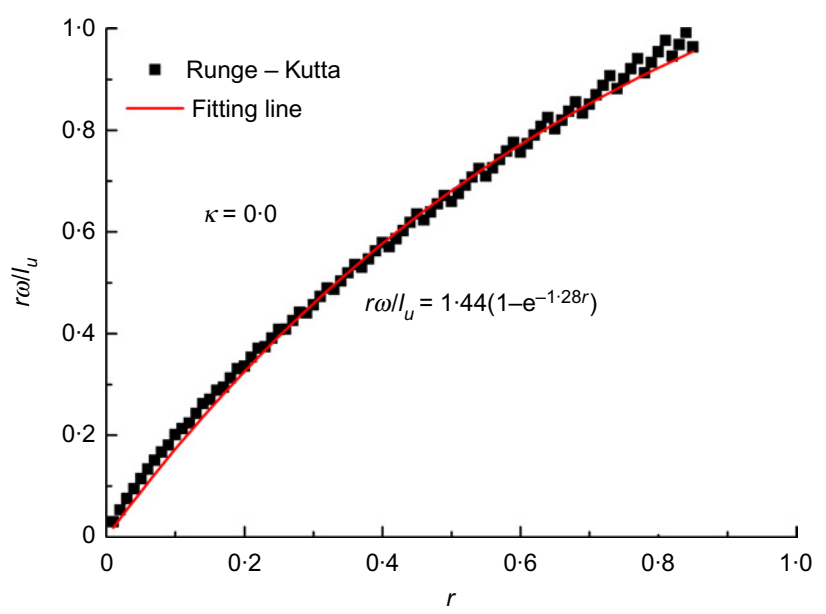

Fig. 15. Influence of the normalised gravity shear stress ratio $r$ on normalised length of process zone (exponential degradation)

Table 5. Values of $A$ and $B$ for equation (55) under different $\boldsymbol{\kappa}$

\begin{tabular}{|c|c|c|c|c|c|c|c|c|}
\hline$\kappa$ & 0 & $0 \cdot 01$ & $0 \cdot 02$ & 0.03 & $0 \cdot 04$ & $0 \cdot 05$ & $0 \cdot 06$ & 0.07 \\
\hline$A$ & 1.44 & $1 \cdot 35$ & $1 \cdot 28$ & 1.23 & $1 \cdot 18$ & $1 \cdot 15$ & $1 \cdot 1$ & $1 \cdot 08$ \\
\hline$B$ & $1 \cdot 28$ & $1 \cdot 31$ & $1 \cdot 35$ & 1.39 & 1.42 & 1.45 & $1 \cdot 51$ & 1.52 \\
\hline$\kappa$ & 0.08 & 0.09 & $0 \cdot 1$ & $0 \cdot 2$ & 0.3 & 0.4 & 0.5 & $0 \cdot 6$ \\
\hline$A$ & 1.04 & $1 \cdot 01$ & 0.99 & 0.77 & $0 \cdot 61$ & 0.49 & $0 \cdot 4$ & $0 \cdot 31$ \\
\hline$B$ & 1.57 & 1.59 & $1 \cdot 61$ & 1.94 & $2 \cdot 35$ & $2 \cdot 8$ & $3 \cdot 4$ & $4 \cdot 45$ \\
\hline
\end{tabular}

$$
l \geq l_{\mathrm{c}}=\left[r_{0} \hat{\omega}+\sqrt{1-r(1-\ln r)}\right] 2 \frac{l_{u}}{r_{0}}
$$

Unlike linear decay, an analytical solution for $\hat{\omega}$ is not possible. Numerical solutions based on differential equations (40) and (41) were achieved in Matlab, using the Runge-Kutta method for $r_{0}=r$. Equations (40) and (41) imply that the values of $\hat{\omega}$ depend on values of $r$ and $\kappa$, as explored below.

First, assume $\kappa=0$, which is satisfied as $l_{u}{ }^{\mathrm{e}} / l_{u} \rightarrow 0$. Fig. 15 plots the value of $r \hat{\omega}$ against the value of $r$. Fitting all the data yields

$$
r \hat{\omega}=1 \cdot 44\left(1-\mathrm{e}^{-1 \cdot 28 r}\right)
$$

Therefore, if $\kappa=0$, the propagation criterion for $r_{0}=r$ would be

$$
l \geq\left[1.44\left(1-\mathrm{e}^{-1 \cdot 28 r}\right)+\sqrt{1-r(1-\ln r)}\right] 2 \frac{l_{u}}{r}
$$

Further it was found that, with a fixed value of $\kappa$, the relationship between $r \hat{\omega}$ and $r$ meets

$$
r \hat{\omega}=A\left(1-\mathrm{e}^{-B r}\right)
$$

where $A$ and $B$ vary with $\kappa$. Table 5 lists values of $A$ and $B$ at typical values of $\kappa$. $A$ and $B$ can be approximately expressed by

$$
A=0 \cdot 84-0 \cdot 87 \kappa+0 \cdot 6 \mathrm{e}^{-10 \kappa}, \quad B=1 \cdot 28+3 \kappa+6 \kappa^{3}
$$

\section{NOTATION}

$A, B$ fitting parameters

$E^{\prime}$ plane strain modulus of sliding layer

$E_{\mathrm{b}}^{\prime} \quad$ plane strain modulus of base layer

$G_{\mathrm{s}}$ shear modulus of shear band material

$g_{\text {acc }}$ accumulated gravitational acceleration

$h, H$ heights of sliding material and overall finite-element model

$l_{u}, l_{u, \mathrm{e}} \quad$ characteristic lengths

$l_{0}, l, \omega, L$ lengths of pre-softened zone, shear band, process zone and overall embankment

$\hat{l}_{0}, \hat{l}, \hat{\omega} \quad$ lengths normalised by $l_{u}$

$P \quad$ lateral force per unit width within the overlying soil
$\hat{P} \quad P$ normalised by product of $l_{u}$ and $\left(\tau_{\mathrm{p}}-\tau_{\mathrm{r}}\right)$

$P_{\mathrm{g}}, P_{\max } \quad$ gravitational and maximum values of $P$

$r, r_{0} \quad$ gravity shear stress ratio outside and inside pre-softened zone

$r_{\mathrm{e}}, r_{0, \mathrm{e}} \quad$ threshold of $r$ and $r_{0}$ from elastic shearing to development of process zone

$S_{\mathrm{t}} \quad$ sensitivity of shear band material

$s$ thickness of shear band

$u$ lateral displacement within overlying soil

$\beta, \kappa \quad$ parameters relating elastic to plastic shear strain ratios between peak and residual strengths

$\gamma$ shear strain within shear band, superscripted by ' $e$ ' and 'p' for elastic and plastic component

$\gamma_{\mathrm{g}}, \gamma_{\mathrm{p}}, \gamma_{\mathrm{r}}$ gravitational, peak and residual values of $\gamma$

$\gamma_{95}^{\mathrm{p}}$ value of $\gamma$ for $95 \%$ degradation of shear strength for exponential decay

$\Delta \tau, \Delta \tau_{\mathrm{r}}, \Delta \tau_{\mathrm{r} 0}, \Delta \tau_{\mathrm{p}}, \Delta P, \quad$ net changes in terms of gravitational $\Delta \delta, \Delta \delta^{\mathrm{e}}, \Delta \delta^{\mathrm{p}}, \Delta \delta_{\mathrm{p}}^{\mathrm{e}}, \Delta u \quad$ values

$\delta$ shear displacement across shear band, superscripted by ' $e$ ' and ' $p$ ' for elastic and plastic component

$\hat{\delta} \quad$ displacements normalised by $\delta_{\mathrm{r}}^{\mathrm{p}}$ or $\delta 9_{5}$

$\bar{\delta} \quad$ characteristic plastic displacement

$\delta_{\mathrm{g}}$ gravitational value of $\delta$

$\delta_{\mathrm{p}}, \delta_{\mathrm{r}} \quad$ peak and residual value of $\delta$ outside pre-softened zone

$\delta_{\mathrm{p} 0}, \delta_{\mathrm{r} 0} \quad$ peak and residual value of $\delta$ inside pre-softened zone

$\delta_{95}^{\mathrm{p}} \quad$ value of $\delta^{\mathrm{p}}$ for $95 \%$ degradation of shear strength for exponential decay

$\theta$ slope angle

$v$ Poisson ratio

$\rho$ submerged density of soil

$\tau$ shear stress within shear band

$\tau_{\mathrm{g}}$ gravitational shear stress

$\tau_{\mathrm{p}}, \tau_{\mathrm{r}}$ peak and residual shear strengths outside pre-softened zone

$\tau_{\mathrm{p} 0}, \tau_{\mathrm{r} 0}$ peak and residual shear strengths within initial pre-softened zone

$\psi$ end-slope angle in finite-element model

\section{REFERENCES}

Andresen, L. \& Jostad, H. P. (2007). Numerical modelling of failure mechanisms in sensitive soft clay - application to offshore geohazards. Proceedings of the offshore technology conference 2007, Houston, TX, paper OTC 18640.

Bernander, S. (2000). Progressive landslides in long natural slopes, formation, potential extension and configuration of finished slides in strain-softening soil. Licentiate thesis, Lulea University of Technology, Lulea, Sweden.

Bernander, S. (2008). Down-hill progressive landslides in soft clays, triggering disturbance agents, slide propagation over horizontal or gentle sloping ground, sensitive related to geometry, Research report. Lulea, Sweden: Department of Civil and Mining Engineering, Lulea University of Technology.

Cecinato, F., Zervos, A. \& Veveakis, E. (2011). A thermomechanical model for the catastrophic collapse of large landslides. Int. J. Numer. Analyt. Methods Geomech. 35, No. 14, $1507-1535$.

Dassault Systèmes (2011). Abaqus version 6.11 documentation. Providence, RI, USA: Dassault Systèmes Simulia Corp.

Einav, I. \& Randolph, M. F. (2005). Combing upper bound and strain path methods for evaluating penetration resistance. Int. J. Numer. Methods Engng 63, No. 14, 1991-2016.

Garagash, D. I. \& Germanovich, L. N. (2012). Nucleation and arrest of dynamic slip on a pressurized fault. J. Geophys. Res. 117, No. B10, paper B10310.

Gray, T., Puzrin, A. M. \& Hill, A. (2015) Application of the shear band propagation method to slope stability analysis 
of a palaeo-landslide in the Caspian Sea. Proceedings of the offshore technology conference, Houston, TX, paper OTC-25869-MS.

Hu, Y. \& Randolph, M. F. (1998). A practical numerical approach for large deformation problem in soil. Int. J. Numer. Analyt. Methods Geomech. 22, No. 5, 327-350.

Locat, A., Leroueil, S., Bernander, S., Demers, D., Jostad, H. P. \& Ouehb, L. (2011). Progressive failure in eastern Canadian and Scandinavian sensitive clays. Can. Geotech. J. 48, No. 11, 1696-1712.

Locat, A., Jostad, H. P. \& Leroueil, S. (2013). Numerical modelling of progressive and its implications for spreads in sensitive clays. Can. Geotech. J. 50, No. 9, 961-978.

Palmer, A. C. \& Rice, J. R. (1973). The growth of slip surface in the progressive failure of over-consolidated clay. Proc. R. Soc. Ser. A: Math. Phys. Sci. 332, No. 1591, 527-548.

Puzrin, A. M. \& Germanovich, L. N. (2005). The growth of shear bands in the catastrophic failure of soils. Proc. R. Soc. Ser. A: Math., Phys. and Engng Sci. 461, No. 2056, 1199-1228.

Puzrin, A. M., Germanovich, L. N. \& Kim, S. (2004). Catastrophic failure of submerged slopes in normally consolidated sediments. Géotechnique 54, No. 10, 631-643, http://dx.doi. org $/ 10.1680 /$ geot.2004.54.10.631.

Puzrin, A. M., Gray, T. \& Hill, A. J. (2015). Significance of the actual non-linear slope geometry for catastrophic failure in submarine landslides. Proc. R. Soc. Ser. A: Math., Phys. and Engng Sci. 471, No. 2175, 20140772.

Quinn, P. E., Diederichs, M. S., Rowe, R. K. \& Hutchinson, D. J. (2011). A new model for large landslides in sensitive clay using a fracture mechanics approach. Can. Geotech. J. 48, No. 8, 1151-1162.

Quinn, P. E., Diederichs, M. S., Rowe, R. K. \& Hutchinson, D. J. (2012). Development of progressive failure in sensitive clay slopes. Can. Geotech. J. 49, No. 7, 782-795.
Rice, J. R. (1968). A path independent integral and the approximate analysis of strain concentration by notches and cracks. J. Appl. Mech. 35, No. 2, 379-386.

Rushton, D., Gray, T. E., Puzrin, A. M. \& Hill, A. J. (2015) GIS-based probabilistic slope stability assessment using shear band propagation. Proceedings of the offshore technology conference, Houston, TX, paper OTC-25871-MS.

Vardoulakis, I. (2000). Catastrophic landslides due to frictional heating of the failure plane. Mech. Cohesive-Frictional Mater. 5, No. 6, 443-467.

Vardoulakis, I. (2002). Dynamic thermo-poro-mechanical analysis of catastrophic landslides. Géotechnique 52, No. 3, 157-171, http://dx.doi.org/10.1680/geot.2002.52.3.157.

Veveakis, M., Stefanou, I. \& Sulem, J. (2013). Failure in shear bands for granular materials: thermo-hydro-chemomechanical effects. Géotechnique Lett. 3, No. 2, 31-36.

Viesca, R. C. \& Rice, J. R. (2012). Nucleation of slip-weakening rupture instability in landslides by localised increase of pore pressure. J. Geophys. Res. 117, No. B3, B03104.

Viesca, R. C., Templeton, E. L. \& Rice, J. R. (2008). Off-fault plasticity and earthquake rupture dynamics: 2 . Effects of fluid saturation. J. Geophys. Res. 113, No. B9, B09307.

Wang, D., Hu, Y. \& Randolph, M. F. (2010). Three-dimensional large deformation finite element analysis of plate anchors in uniform clay. J. Geotech. Geoenviron. Engng ASCE 136, No. 2, 355-365.

Wang, D., Gaudin, C. \& Randolph, M. F. (2013a). Large deformation finite element analysis investigating the performance of anchor keying flap. Ocean Engng 59, 107-116.

Wang, D., Randolph, M. F. \& White, D. J. (2013b). A dynamic large deformation finite element method based on mesh regeneration. Comput. Geotech. 54, 192-201.

Wang, D., Bienen, B., Nazem, M., Tian, Y., Zheng, J., Pucker, T. \& Randolph, M. F. (2015). Large deformation finite element analyses in geotechnical engineering. Comput. Geotech. 65, $104-114$. 